J. Nonlinear Var. Anal. 2 (2018), No. 2, pp. 233-262

Available online at http://jnva.biemdas.com

https://doi.org/10.23952/jnva.2.2018.2.10

\title{
HOPF BIFURCATION OF RELATIVE PERIODIC SOLUTIONS IN A SYSTEM OF FIVE PASSIVELY MODE-LOCKED LASERS
}

\author{
ZALMAN BALANOV ${ }^{2,1, *}$, PAVEL KRAVETC ${ }^{2}$, WIESLAW KRAWCEWICZ ${ }^{1,2}$, DMITRII RACHINSKII $^{2}$, HAO-PIN WU $^{2}$ \\ ${ }^{1}$ Center for Applied Mathematics at Guangzhou University, Guangzhou 510006, China \\ ${ }^{2}$ Department of Mathematical Sciences, The University of Texas at Dallas, Richardson 75080, USA
}

\begin{abstract}
The goal of this paper is to study the equivariant Hopf bifurcation of relative periodic solutions from relative equilibria in a system of five identical passively mode-locked semiconductor lasers coupled in the $S_{5}$-equivariant fashion. Each laser is described by a delay differential model respecting a spacial $S^{1}$-symmetry. The existence of branches of relative periodic solutions together with their symmetric classification is established using the equivariant twisted $S_{5} \times S^{1}$-degree with one free parameter. Theoretical results are supported by numerical simulations.

Keywords. Functional differential equation; Symmetric coupling; Equivariant Hopf bifurcation; Equivariant degree method; Relative periodic solution.
\end{abstract}

2010 Mathematics Subject Classification. 34K18, 37N20.

\section{INTRODUCTION}

The analysis of Hopf bifurcation of periodic solutions from an equilibrium state (both in non-equivariant and equivariant setting) has been done by many authors using different techniques. A natural counterpart of an equilibrium state in dynamical systems with continuous symmetries is a relative equilibrium, i.e. an equilibrium modulo the group action. Similarly, a counterpart of a periodic solution is a relative periodic solution. In particular, in $S^{1}$-symmetric systems, the $S^{1}$-equivariant Hopf bifurcation is responsible for branching of relative periodic solutions from a relative equilibrium. This scenario is analogous to the classical Hopf bifurcation of periodic solutions from an equilibrium state in generic systems (without symmetry).

Relative equilibrium states and relative periodic motions are well-known for conservative systems related to rigid bodies [34], deformable bodies [13], molecular vibrations [28], celestial mechanics $[12,35]$ and vortex theory[39] (see also $[19,53]$ and references therein). In addition to $S^{1}$-symmetry, many of such systems respect a finite group $\Gamma$ of spatial symmetries such as, for example, a symmetry of coupling of atoms in molecules [37]. This naturally leads to the problem of classification of relative equilibria/relative periodic solutions according to their symmetric properties (spatio-temporal symmetries) represented by a subgroup $H$ of the group $\Gamma \times S^{1}$ for relative equilibria and the group $\Gamma \times S^{1} \times S^{1}$

\footnotetext{
${ }^{*}$ Corresponding author.

E-mail addresses: balanov@utdallas.edu (Z. Balanov), pavel.kravetc@utdallas.edu (P. Kravetc), wieslaw@utdallas.edu (W. Krawcewicz), Dmitry.Rachinskiy@utdallas.edu (D. Rachinskii), Hao-pin.Wu@utdallas.edu (H. Wu).

Received April 7, 2018; Accepted June 13, 2018.
}

(C)2018 Journal of Nonlinear and Variational Analysis 
for relative periodic solutions, respectively, where the second copy of $S^{1}$ is associated with time periodicity. Examples include dynamics of a deformable body in an ideal irrotational fluid [53], symmetric celestial motions, for instance, central configurations [36, 40], etc. On the other hand, there is a long list of applications described by non-conservative systems of DEs admitting relative equilibria/relative periodic solutions (see, for example, [19], where the Couette-Taylor experiment is discussed in detail).

In his pioneering work [30] (cf. [18]), M. Krupa proposed a general method for analysis of the bifurcation of relative periodic solutions from a relative equilibrium for systems of ordinary differential equations (in general, non-Hamiltonian). This elegant method reduces the problem to the analysis of a generic (non-symmetric) Hopf bifurcation for an explicit differential equation on the normal slice to the relative equilibrium and related normal form classification. An extension of Krupa's method to the case of more complicated spatial symmetries (including $\Gamma \times S^{1}$ ) has been developed in [48] (see also [14] for the moving frames method and [31] for the hierarchy of secondary bifurcations). Although, in principal, Krupa's method can be adapted for analysis of FDEs, we are not aware of any published work in this direction.

On the other hand, an equivariant degree based method allowing to study the occurrence of Hopf bifurcation of relative equilibria/relative periodic solutions in FDEs together with their symmetric properties, was suggested in [4]. This method is equally applicable to smooth and non-smooth setting (assuming differentiability at selected points; cf. [6], where a system of coupled van der Pol oscillators was considered in the presence of hysteretic non-linearities). Also, it is insensitive to violations of genericity assumptions $[16,22]$ (including the simplicity of purely imaginary eigenvalues at the bifurcation point, transversality of the eigenvalue crossing, and non-resonance conditions). At the same time, this method does not allow to obtain stability results for bifurcating branches. For a detailed exposition of the equivariant degree theory and its applications, we refer to $[5,7,8,9,23,24,29])$. The goal of the present paper is to apply the abstract results from [4] to study equivariant Hopf bifurcation of relative equilibria/relative periodic solutions in delay differential equations (DDE) describing a system of 5 mode-locked lasers coupled in $S_{5}$-symmetric fashion. The rate equations of semiconductor lasers are $S^{1}$-symmetric (see, for example, $[15,17,54])$ and can naturally include delays, the classical example being the Lang-Kabayashi model where the light is re-injected into the laser cavity by an external mirror $[32,55]$.

Semiconductor mode locked lasers are compact reliable low-cost devices that emit short optical pulses at high repetition rates suitable for applications in telecommunications, machining, probing, all optical systems, etc. [50]. We use a delay differential model of a passively mode-locked laser proposed in [51]. This delay differential system has been extensively applied to analyze instabilities $[38,41]$ and hysteresis [21, 43] in mode-locked lasers, optically injected lasers [2, 45], hybrid mode locking [3], noise reduction [25], resonance to delayed feedback [1], and Fourier domain mode locking [46]. The same system has been used to model four identical lasers coupled in $D_{4}$-symmetric fashion [44]. An increasing interest in small systems of symmetrically coupled lasers and large synchronized laser arrays motivates the analysis of other symmetric configurations of coupled lasers [26, 33, 42, 49].

In the mode-locking regime, the laser emits a periodic sequence of light pulses with the period close to the cold cavity round trip time, which equals to the delay in the model. A typical bifurcation scenario associated with formation of this regime is the Hopf bifurcation of a relative equilibrium (continuous wave solution) from the "laser off" equilibrium followed by the $S^{1}$-equivariant Hopf bifurcation of a relative periodic solution from the relative equilibrium with the increase of the bifurcation parameter (pump 
current). As the bifurcation parameter increases further, the relative periodic solution continuously transforms to acquire a pulsating shape. This transformation is simultaneous with a sequence of secondary Hopf bifurcations from the equilibrium and relative equilibrium solutions.

After the Introduction, the paper is organized as follows. In Section 2, we recall main abstract results from [4] on the equivariant Hopf bifurcation of relative equilibria/relative periodic solutions (see Proposition 2.1 and Theorem 2.1). In Section 3, these results are applied to delay rate equations of the $S_{5} \times S^{1}$-symmetric laser system. We prove the occurrence of infinitely many branches of relative equilibria with various symmetries from the laser off state. Then, the analytic method is combined with numerical computations to analyze symmetric properties of relative periodic solutions that branch from the relative equilibrium states. We complete the paper with two appendices: Section A lists a few twisted subgroups, which are used in Section 3 to describe symmetries of solutions, and recalls the standard equivariant jargon; in Section B, we formulate a conjecture on the reduced system related to nonzero components of a relative equilibrium.

\section{2. $\Gamma \times S^{1}$-Symmetric Systems of FDEs: Abstract FrAmEWORK}

2.1. Notation and statement of the problem. In what follows, $\Gamma$ stands for a finite group and $V:=\mathbb{R}^{n}$ denotes an orthogonal $\Gamma \times S^{1}$-representation with the $S^{1}$-action given by the homomorphism $T: S^{1} \rightarrow$ $O(n)$. Set

$$
J:=\lim _{\tau \rightarrow 0} \frac{1}{\tau}\left[T\left(e^{i \tau}\right)-\mathrm{Id}\right]
$$

For all $e^{i \tau} \in S^{1}$, one has:

$$
\forall_{v \in V} \quad e^{i \tau} v=e^{\tau J}(v), \quad J e^{\tau J}=e^{\tau J} J
$$

Denote $\mathscr{G}:=\Gamma \times S^{1}$ and set:

$$
\mathbf{\square}:=\Gamma \times\{1\} \quad \text { and } \quad \mathbf{S}:=\{e\} \times S^{1},
$$

where $e \in \Gamma$ is the neutral element. The $\mathbf{S}$-isotypical decomposition of $V$ reads

$$
V=V_{0} \oplus V_{1} \oplus \cdots \oplus V_{m},
$$

where $V_{0}$ corresponds to the trivial action while $V_{k}$ with $k>0$ is modeled on the $S^{1}$-irreducible representation $\mathscr{V}_{k} \simeq \mathbb{C}$ with the $S^{1}$-action given by $e^{i \tau} z:=e^{i k \tau} \cdot z$, where '.' stands for the complex multiplication. Also, $J v=i k \cdot v$ for $v \in V_{k},(k \geq 0)$.

Let $r>0$ and denote by $C_{-r}(V)$ the Banach space

$$
C([-r, 0] ; V):=\{x: \text { where } x:[-r, 0] \rightarrow V \text { is a continuous map }\},
$$

equipped with the norm $\|x\|_{\infty}:=\sup \{|x(\theta)|: \theta \in[-r, 0]\}$. Clearly, $C_{-r}(V)$ is an isometric $\mathscr{G}$-representation with the action given by

$$
\forall_{\theta \in[-r, 0]}\left(\left(\gamma, e^{i \tau}\right) x\right)(\theta)=e^{\tau J}(\gamma x(\theta)), x \in C_{-r}(V), \quad\left(\gamma, e^{i \tau}\right) \in \mathscr{G} .
$$

Decomposition (2.1) induces the $\mathbf{S}$-decomposition

$$
C_{-r}(V)=\bigoplus_{k=0}^{m} C_{-r}\left(V_{k}\right)
$$


For a continuous map $x: \mathbb{R} \rightarrow V$ and $t \in \mathbb{R}$, let $x_{t}:[-r, 0] \rightarrow V$ be a map defined by

$$
x_{t}(\theta):=x(t+\theta), \quad \theta \in[-r, 0] .
$$

We make the following assumption:

(A0) $f: \mathbb{R} \times C_{-r}(V) \rightarrow V$ is a $\mathscr{G}$-equivariant map, i.e. for $\left(\gamma, e^{i \tau}\right) \in \mathscr{G}$

$$
f\left(\alpha,\left(\gamma, e^{\tau i}\right) x\right)=e^{\tau J} \gamma f(\alpha, x) \quad \text { for all } x \in C_{-r}(V) .
$$

Consider the parametrized system of FDEs

$$
\dot{x}(t)=f\left(\alpha, x_{t}\right), \quad x(t) \in V .
$$

In what follows, we are interested in bifurcations of continuous branches of periodic/quasi-periodic solutions to (2.3) of special type as well as in their symmetric properties.

2.2. Symmetric bifurcation of relative equilibria from an equilibrium. In this subsection, we describe one result on the equivariant Hopf bifurcation of periodic solutions to (2.3) of the type

$$
x(t)=e^{w J t} x \quad \text { for some } x \in V \text { and } w \in \mathbb{R} .
$$

2.2.1. Relative equilibria. Take decomposition (2.1), where $V_{0}=V^{\mathbf{S}}$, and denote

$$
V_{*}:=V_{0}^{\perp}=V_{1} \oplus \cdots \oplus V_{m} .
$$

For a fixed $\lambda=u+i w \in \mathbb{C}$, define the linear operator $\xi(\lambda): V \rightarrow C_{-r}(V)$ by

$$
(\xi(\lambda) x)(\theta)=e^{u \theta} e^{w J \theta} x_{*}+x_{o}, \quad \theta \in[-r, 0],
$$

where $x=x_{*}+x_{o}, x_{*} \in V_{*}$ and $x_{o} \in V_{0}$. Take the map $\tilde{f}: \mathbb{R} \times \mathbb{C} \times V \rightarrow V$ defined by

$$
\widetilde{f}(\alpha, \lambda, x):=f(\alpha, \xi(\lambda) x)
$$

and consider the equation

$$
w J x=\widetilde{f}(\alpha, i w, x), \quad x \in V .
$$

Recall the following standard definition (see, for example, [19]).

Definition 2.1. Suppose that (2.7) holds for some $\alpha_{o}, w_{o} \in \mathbb{R}$ and $x_{o} \notin V^{\mathbf{S}}$. Then, the orbit $\mathbf{S}\left(x_{o}\right)$ is a one-dimensional curve in $V$ called a relative equilibrium of equation (2.3). For $w_{o} \neq 0$, this orbit is a trajectory of time-periodic solutions $x(\cdot)=e^{\left(w_{o} \cdot+\tau\right) J} x_{o}, e^{i \tau} \in S^{1}$, to equation (2.3) called a rotating wave.

2.2.2. Characteristic quasi-polynomials. In what follows, we will require from $f$ to satisfy minimal differentiability properties, i.e. it will be assumed that once we use the symbols of derivatives of $f$, it is well-defined on a specified set.

Let $\alpha_{o} \in \mathbb{R}$ be given and let $x_{o} \in V^{\mathscr{G}}$ be an equilibrium for (2.3). We will also call the pair $\left(\alpha_{o}, x_{o}\right)$ an equilibrium, or a stationary solution, in this case.

Let us consider the bifurcation of relative equilibria from this equilibrium. Denote by

$$
D_{\mathbf{x}} f(\alpha, \mathbf{x}): \mathbb{R} \times C_{-r}(V) \rightarrow V
$$

the derivative of the map $f$ with respect to $\mathbf{x} \in C_{-r}(V)$ (provided that this derivative exists). If $x_{o} \in V_{0}$, then the Jacobi matrix $D_{x} \widetilde{f}\left(\alpha, \lambda, x_{o}\right): V \rightarrow V$, which is given by

$$
D_{x} \widetilde{f}\left(\alpha, \lambda, x_{o}\right)=D_{\mathbf{x}} f\left(\alpha, \xi(\lambda) x_{o}\right) \xi(\lambda)
$$


is $\mathbf{S}$-equivariant (cf. (2.5) and (2.6)). Therefore, the subspaces $V_{0}$ and $V_{*}$ are $\mathbf{S}$-invariant for this matrix. Consider the restrictions $\left.D_{x} \widetilde{f}\left(\alpha, \lambda, x_{o}\right)\right|_{V_{0}}$ and $\left.D_{x} \widetilde{f}\left(\alpha, \lambda, x_{o}\right)\right|_{V_{*}}$ and define the characteristic quasipolynomials for $x_{o} \in V_{0}$ and $\lambda \in \mathbb{C}$ :

$$
\begin{aligned}
& \mathscr{P}_{0}\left(\alpha, \lambda, x_{o}\right):=\operatorname{det}_{\mathbb{C}}\left(\left.D_{x} \widetilde{f}\left(\alpha, \lambda, x_{o}\right)\right|_{V_{0}}-\lambda \mathrm{Id}\right), \\
& \mathscr{P}_{*}\left(\alpha, \lambda, x_{o}\right):=\operatorname{det}_{\mathbb{C}}\left(\left.D_{x} \widetilde{f}\left(\alpha, \lambda, x_{o}\right)\right|_{V_{*}}-\lambda \mathrm{Id}\right) .
\end{aligned}
$$

Assume that:

(A1) (a) There exists a continuous function $x:\left(\alpha_{o}-\varepsilon, \alpha_{o}+\varepsilon\right) \rightarrow V_{0}$ (for some $\varepsilon>0$ ) such that: $x\left(\alpha_{o}\right)=x_{o} \in V^{\mathscr{G}}$ and $\left\{(\alpha, x(\alpha)): \alpha \in\left(\alpha_{o}-\varepsilon, \alpha_{o}+\varepsilon\right)\right\}$ consists of equilibria for (2.3);

(b) $D_{\mathbf{x}} f(\alpha, x(\alpha))$ exists for $\alpha \in\left(\alpha_{o}-\varepsilon, \alpha_{o}+\varepsilon\right)$ and depends continuously on $\alpha$;

(c) $\mathscr{P}_{0}(\alpha, 0, x(\alpha)) \neq 0$.

Definition 2.2. Let $x$ be as in condition (A1). The set $M \subset \mathbb{R}^{2} \times V^{\mathscr{G}}$ given by

$$
M:=\left\{(\alpha, w, x(\alpha)): \alpha \in\left(\alpha_{o}-\varepsilon, \alpha_{o}+\varepsilon\right), w \in \mathbb{R}\right\}
$$

is called the set of trivial solutions to (2.7).

Assume that:

(A2) The quasi-polynomial $\mathscr{P}_{*}\left(\alpha_{o}, \cdot, x_{o}\right)$ has a characteristic root $\lambda=i w_{o}$ for some $w_{o} \in \mathbb{R}$ at the equilibrium point $\left(\alpha_{o}, x_{o}\right)$, but for any other equilibrium $(\alpha, x)$ from a neighborhood of $\left(\alpha_{o}, x_{o}\right)$ in $\mathbb{R} \times V_{0}$, the corresponding characteristic polynomial has no roots of the form $\lambda=i w, w \in \mathbb{R}$.

In order to find nontrivial solutions to (2.7) bifurcating from $M$, consider the equation

$$
\Phi(\alpha, w, x):=\widetilde{f}(\alpha, i w, x)-w J x=0
$$

as a $\mathscr{G}$-symmetric bifurcation problem with two free parameters $\alpha$ and $w$. Assumption (A2) implies that $D_{x} \widetilde{f}\left(\alpha_{o}, i w_{o}, x_{o}\right)-w_{o} J: V \rightarrow V$ is not an isomorphism (i.e. (A2) provides a necessary condition for the bifurcation of relative equilibria), while assumption (A1)(c) prevents the steady-state bifurcation.

2.2.3. Sufficient condition for bifurcation of relative equilibria. Take the $\mathscr{G}$-isotypical decomposition of $V(\operatorname{see}(2.1))$ :

$$
V=V_{0} \oplus V_{*}=\bigoplus_{i} V_{i}^{0} \oplus \bigoplus_{j, k} V_{j, k} \quad(i=0, \ldots, r ; j=0, \ldots, s ; k=1, \ldots, m),
$$

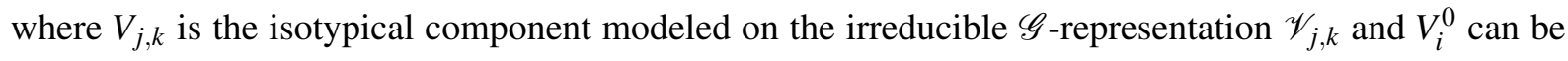
identified with the $\mathbf{m}$-representation modeled on the irreducible $\mathbf{m}$-representation $\mathscr{V}_{i}$. For any $j=0, \ldots, s$ and $k=1, \ldots, m$, put

$$
\mathscr{P}_{j, k}(\alpha, \lambda):=\operatorname{det}_{\mathbb{C}}\left(\left.D_{x} \widetilde{f}(\alpha, \lambda, x(\alpha))\right|_{V_{j, k}}-\lambda \mathrm{Id}\right), \quad \lambda \in \mathbb{C} .
$$

Notice that the characteristic equation at $(\alpha, x(\alpha))$ can be written as

$$
\mathscr{P}_{*}(\alpha, \lambda):=\mathscr{P}_{*}(\alpha, \lambda, x(\alpha))=\prod_{k>0} \prod_{j=0}^{s} \mathscr{P}_{j, k}(\alpha, \lambda)=0 .
$$

This implies that $\lambda$ is a characteristic root for $(\alpha, x(\alpha))$ if it is a root of $P_{j, k}(\alpha, \lambda)=0$ for some $k>0$ and $j \geq 0$. 
To formulate our first bifurcation result, we need two additional concepts. Observe that using (A2), one can choose a small neighborhood $Q$ of the point $i w_{o}$ in the right half-plane $\operatorname{Re} \lambda>0$ of $\mathbb{C}$ and a sufficiently small real $\delta=\delta(Q)>0$ such that, as $\alpha$ varies over the interval $\left|\alpha-\alpha_{o}\right| \leq \delta$, the roots $\lambda(\alpha)$ of $\mathscr{P}_{j, k}(\alpha, \cdot)$ can only leave $Q$ through the 'exit' at the point $i w_{o}$ and only when $\alpha=\alpha_{o}$.

Definition 2.3. Define the $V_{j, k}$-isotypical crossing number at $\left(\alpha_{o}, w_{o}\right)$ by the formula

$$
\mathfrak{t}_{j, k}\left(\alpha_{o}, w_{o}\right):=\mathfrak{t}_{j, k}^{-}\left(\alpha_{o}, w_{o}\right)-\mathfrak{t}_{j, k}^{+}\left(\alpha_{o}, w_{o}\right),
$$

where $\mathfrak{t}_{j, k}^{-}\left(\alpha_{o}, w_{o}\right)$ is the number of roots $\lambda(\alpha)$ of $\mathscr{P}_{j, k}(\alpha, \cdot)$ (counted according to their $\mathscr{V}_{j, k}$-isotypical multiplicity) in the set $Q$ for $\alpha<\alpha_{o}$, and $\mathfrak{t}_{j, k}^{+}\left(\alpha_{o}, w_{o}\right)$ is the number of roots of $\mathscr{P}_{j, k}(\alpha, \cdot)$ in $Q$ for $\alpha>\alpha_{o}$.

Definition 2.4. A set $K$ of solutions $(\alpha, w, x)$ to equation (2.7) is called a continuous branch of relative equilibria bifurcating from the equilibrium $\left(\alpha_{o}, x_{o}\right)$ of equation (2.3) if:

(i) $x \notin V^{\mathbf{S}}$ for all $(\alpha, w, x) \in K$;

(ii) $\bar{K}$ contains a connected component $K_{o}$ such that $K_{o} \cap M \neq \emptyset$ (cf. (2.11));

(iii) For any $\varepsilon>0$, there is a $\delta>0$ such that if $(\alpha, w, x) \in K \cap K_{o}$ and $\|x\|<\delta$, then $\left|\alpha-\alpha_{o}\right|<\varepsilon$ and $\left|w-w_{o}\right|<\varepsilon$.

A sufficient condition for the bifurcation of relative equilibria from the equilibrium $\left(\alpha_{o}, x_{o}\right)$, which provides an estimate for the number of possible branches of relative equilibria with their symmetric properties, can be formulated as follows.

Proposition 2.1 (see [4]). Given system (2.3), assume conditions (A0)-(A2) are satisfied. Let ( $\left.\mathscr{H}_{0}\right)$ be a maximal twisted orbit type in $V$. Take decomposition (2.13) and denote by $\mathfrak{M}$ the set of all $\mathscr{G}$-isotypical components in which $\left(\mathscr{H}_{o}\right)$ is an orbit type. Assume there exists $V_{j_{o}, k_{o}} \in \mathfrak{M}$ such that:

(i) $\left(\mathscr{H}_{o}\right)$ is a maximal twisted type in $V_{j_{o}, k_{o}}$;

(ii) $\mathfrak{t}_{j_{o}, k_{o}}\left(\alpha_{o}, w_{o}\right) \neq 0$ (cf. (2.15));

(iii) $\mathfrak{t}_{j, k}\left(\alpha_{o}, w_{o}\right) \cdot \mathfrak{t}_{j^{\prime}, k^{\prime}}\left(\alpha_{o}, w_{o}\right) \geq 0 \quad$ for all $V_{j, k}, V_{j^{\prime}, k^{\prime}} \in \mathfrak{M}$.

Then, there exist at least $\left.|\mathscr{G}| \mathscr{H}_{o}\right|_{\mathbf{S}}$ continuous branches of relative equilibria of equation (2.3) bifurcating from the equilibrium $\left(\alpha_{o}, x_{o}\right)$ with the minimal symmetry $\left(\mathscr{H}_{o}\right)$ (here $|\cdot| \mathbf{S}$ stands for the number of $\mathbf{S}$ orbits).

\subsection{Hopf bifurcation from a relative equilibrium.}

2.3.1. Regular relative equilibria. Suppose that for some $\alpha=\bar{\alpha}$ and $\bar{x} \in V \backslash V^{\mathbf{S}}$, equation (2.3) has a relative equilibrium $\mathbf{S}(\bar{x})$ (see Definition 2.1). Then, equation (2.7) is satisfied for some $\bar{w} \in \mathbb{R}$ :

$$
\Phi(\bar{\alpha}, \bar{w}, \bar{x})=\widetilde{f}(\bar{\alpha}, i \bar{w}, \bar{x})-\bar{w} J \bar{x}=0,
$$

where $\bar{x}=(\bar{q}, \bar{v}) \in V^{\mathbf{S}} \oplus V_{*}$ with $\bar{v} \neq 0$. Moreover,

$$
\left.\frac{d}{d \tau} \Phi\left(\bar{\alpha}, \bar{w}, e^{\tau J} \bar{x}\right)\right|_{\tau=0}=\left.D_{x} \Phi\left(\bar{\alpha}, \bar{w}, e^{\tau J} \bar{x}\right) J e^{\tau J} \bar{x}\right|_{\tau=0}=D_{x} \Phi(\bar{\alpha}, \bar{w}, \bar{x}) J \bar{x}
$$

provided that the derivatives in (2.17) exist. Hence, relation (2.16) and the $\mathbf{S}$-equivariance of $\Phi$ imply that the directional derivative of $\Phi$ at the point $\bar{x}$ in the direction of the orbit $\mathbf{S}(\bar{x})$ is zero:

$$
D_{x} \Phi(\bar{\alpha}, \bar{w}, \bar{x}) J \bar{x}=0 .
$$

That is, the map $D_{x} \Phi(\bar{\alpha}, \bar{w}, \bar{x})$ has a non-zero kernel. 
Definition 2.5. A relative equilibrium $\mathbf{S}(\bar{x})$ will be called regular if the kernel of the map given by the following block-matrix

$$
\left[D_{w} \Phi(\bar{\alpha}, \bar{w}, \bar{x}) \mid D_{x} \Phi(\bar{\alpha}, \bar{w}, \bar{x})\right]: \mathbb{R} \times V \rightarrow V
$$

is one-dimensional, provided that the derivatives in (2.19) exist.

We make the following assumption parallel to (A1):

(A3) (a) Given $(\bar{\alpha}, \bar{w}, \bar{x})$ satisfying (2.16), assume that there exist a neighborhood $\mathfrak{U}$ of $\bar{\alpha}$ in $\mathbb{R}$ and continuous functions $w: \mathfrak{U} \rightarrow \mathbb{R}, w(\bar{\alpha})=\bar{w}$, and $u: \mathfrak{U} \rightarrow S:=\{x \in V: x \bullet J \bar{x}=0\}, u(\bar{\alpha})=0$, such that

$$
\Phi(\alpha, w(\alpha), \bar{x}+u(\alpha))=0, \quad \alpha \in \mathfrak{U}
$$

i.e., formula

$$
x_{\alpha}(t):=e^{(w(\alpha) t+\tau) J} x(\alpha)
$$

defines a branch of relative equilibria $\mathbf{S}(x(\alpha))$ parametrized by $\alpha \in \mathfrak{U}$ (here $x(\alpha)=\bar{x}+u(\alpha)$ );

(b) $D_{\mathbf{x}} f\left(\alpha, x_{\alpha}(\cdot)\right)$ exists for $\alpha \in\left(\alpha_{o}-\varepsilon, \alpha_{o}+\varepsilon\right)$ and depends continuously on $\alpha$;

(c) $\mathbf{S}(x(\alpha))$ is a regular relative equilibrium for (2.3) for all $\alpha \in \mathfrak{U}$.

It will be assumed that the branch $\mathbf{S}(x(\alpha))$ has symmetric properties (cf. Proposition 2.1):

(A4) The regular relative equilibrium $\mathbf{S}(\bar{x}), \alpha \in \mathfrak{U}$, admits a twisted group symmetry $\mathscr{H}<\mathscr{G}$.

For a given $\alpha \in \mathfrak{U}$, put

$$
\mathscr{B}_{\alpha}:=D_{\mathbf{x}} f\left(\alpha, e^{w(\alpha) J} x(\alpha)\right)=D_{\mathbf{x}} f(\alpha, \xi(i w(\alpha)) x(\alpha)): C_{-r}(V) \rightarrow V .
$$

For $\alpha \in \mathfrak{U}$ and $\lambda \in \mathbb{C}$, define the linear map $\mathscr{R}_{\alpha}: V^{c} \rightarrow V^{c}$ in the complexification $V^{c}$ of $V$ by the formula

$$
\mathscr{R}_{\alpha}(\lambda) y:=\mathscr{B}_{\alpha}\left(e^{(w(\alpha) J+\lambda \mathrm{Id})} \cdot y\right), \quad y \in V^{c}
$$

Then,

$$
\operatorname{det}_{\mathbb{C}}\left(\mathscr{R}_{\alpha}(\lambda)-w(\alpha) J-\lambda \text { Id }\right)=0
$$

is the characteristic equation for the linearization of system (2.3) on the relative equilibrium $\mathbf{S}(x(\alpha))$. Since

$$
\mathscr{R}_{\alpha}(0)-w(\alpha) J=D_{x} \Phi(\alpha, w(\alpha), x(\alpha)),
$$

conditions (A3)(a,b) imply that the characteristic equation (2.24) has a zero root $\lambda=0$ corresponding to the eigenvector $J x(\alpha)$; furthermore, due to (A3)(c), this root is simple.

2.3.2. Hopf bifurcation of relative periodic solutions. We are interested in finding solutions to (2.3) of the form

$$
x(t)=e^{(w(\alpha)+\phi) t J}(x(\alpha)+y(t)),
$$

where $y(t)$ is a non-stationary $p$-periodic function with $p=2 \pi / \beta$ for some $\beta>0$, and in symmetric properties of these solutions. Here $y(t)$ and $\beta, \phi \in \mathbb{R}$ are unknown. Periodic and quasi-periodic solutions of type (2.26) are called relative periodic solutions.

To be more precise, let us define the so-called equivariant Hopf bifurcation of small amplitude relative periodic solutions of type (2.26) from the family of relative equilibria (2.21).

The following definition is similar to Definition 2.4. 
Definition 2.6. A set $K$ of quadruplets $(\alpha, \beta, \phi, x)$, where $x$ is a solution to equation (2.3) of the form (2.26), is called a continuous branch of relative periodic solutions bifurcating (via the equivariant Hopf bifurcation) from the relative equilibrium $(\bar{\alpha}, \bar{w}, \mathbf{S}(\bar{x}))$ if there exists a $\beta_{o}>0$ such that:

(i) $\bar{K}$ contains a connected component $K_{o}$ such that $\left(\bar{\alpha}, \beta_{o}, 0, \bar{x}\right) \in K_{o}$;

(ii) For any $\varepsilon>0$, there is a $\delta>0$ such that if $(\alpha, \beta, \phi, x) \in K \cap K_{o}$ and $\|y\|<\delta$, then $|\alpha-\bar{\alpha}|<\varepsilon$, $|\phi|<\delta$, and $\left|\beta-\beta_{o}\right|<\varepsilon$.

A necessary condition for the Hopf bifurcation is that characteristic equation (2.24) has a pair of purely imaginary roots $\lambda= \pm i \beta_{o}, \beta_{o}>0$, for $\alpha=\bar{\alpha}$. We make a stronger assumption:

(A5) Characteristic equation (2.24) has a pair of purely imaginary roots $\lambda= \pm i \beta_{o}, \beta_{o}>0$, for $\alpha=\bar{\alpha}$, and has no roots of the form $\lambda=i \beta, \beta \geq 0$, for $\alpha \neq \bar{\alpha}, \alpha \in \mathfrak{U}$.

Put $\mathscr{K}:=\mathscr{H} \times S^{1}$ and consider the $\mathscr{K}$-isotypical decomposition of $V^{c}$ :

$$
V^{c}=U_{0,1} \oplus U_{1,1} \oplus \cdots \oplus U_{p, 1},
$$

where $S^{1}$-action is given by complex multiplication. Due to the equivariance, each isotypical component $U_{j, 1}$ is invariant for the map $\mathscr{R}_{\alpha}(\lambda)$ (see (2.23)) and for $J$. Therefore, we can introduce the characteristic polynomial

$$
\overline{\mathscr{P}}_{j, 1}(\alpha, \lambda):=\operatorname{det}_{\mathbb{C}}\left(\left.\left(\mathscr{R}_{\alpha}(\lambda)-w(\alpha) J-\lambda \operatorname{Id}\right)\right|_{U_{j, 1}}\right), \quad \lambda \in \mathbb{C},
$$

associated with each isotypical component $U_{j, 1}$, and define the $U_{j, 1}$-isotypical crossing numbers

$$
\overline{\mathfrak{t}}_{j, 1}\left(\bar{\alpha}, \beta_{o}\right)=\overline{\mathfrak{t}}_{j, 1}^{-}\left(\bar{\alpha}, \beta_{o}\right)-\overline{\mathfrak{t}}_{j, 1}^{+}\left(\bar{\alpha}, \beta_{o}\right)
$$

at the point $\left(\bar{\alpha}, \beta_{o}\right)$ in the same way as we did in Subsection 2.2 (cf. (2.15)).

Theorem 2.1 (see [4])). Given system (2.3), assume conditions (A0) and (A3)-(A5) are satisfied. Take decomposition (2.27) and let $\left(\mathscr{L}_{o}\right)$ be a maximal twisted orbit type in $V^{c}$. Denote by $\mathfrak{N}$ the set of all $\mathscr{K}$-isotypical components in (2.27) in which $\left(\mathscr{L}_{o}\right)$ is an orbit type. Assume there exists $U_{j_{o}, 1} \in \mathfrak{N}$ such that:

(i) $\left(\mathscr{L}_{o}\right)$ is a maximal twisted orbit type in $U_{j_{o}, 1}$;

(ii) $\mathfrak{t}_{j_{o}, 1}\left(\alpha_{o}, w_{o}\right) \neq 0(c f .(2.15))$;

(iii) $\mathfrak{t}_{j, 1}\left(\alpha_{o}, w_{o}\right) \cdot \mathfrak{t}_{j^{\prime}, 1}\left(\alpha_{o}, w_{o}\right) \geq 0 \quad$ for all $U_{j, 1}, U_{j^{\prime}, 1} \in \mathfrak{N}$.

Then, there exist at least $\left|\mathscr{K} / \mathscr{L}_{o}\right|_{S^{1}}$ continuous branches of relative periodic solutions (2.26) bifurcating via the Hopf bifurcation from the relative equilibrium $(\bar{\alpha}, \bar{w}, \mathbf{S}(\bar{x}))(c f$. Definition 2.6) and having the minimal symmetry $\left(\mathscr{L}_{o}\right)$.

\section{Dde Model of a $S_{5}$-Configuration of Passively Mode-Locked Semiconductor}

\section{LASERS}

3.1. Mathematical model. In [52], a model for a mode-locked semiconductor laser with gain and absorber sections was introduced as a system of the following delay differential equations:

$$
\left\{\begin{array}{l}
\dot{g}(t)=g_{0}-\gamma_{g} g(t)-\frac{1}{E_{g}} e^{-q(t)}\left(e^{g(t)}-1\right)|a(t)|^{2}, \\
\dot{q}(t)=q_{0}-\gamma_{q} q(t)-\frac{1}{E_{q}}\left(1-e^{-q(t)}\right)|a(t)|^{2} \\
\dot{a}(t)=-\gamma a(t)+\gamma \sqrt{\kappa} \exp \left[\frac{\left(1-i \eta_{g}\right) g(t-T)-\left(1-i \eta_{q}\right) q(t-T)}{2}\right] a(t-T) .
\end{array}\right.
$$


The complex-valued function $a(t)$ is the field amplitude at the entrance of the absorber section with $|a(t)|^{2}$ representing the optical power. The real-valued functions $g(t)$ and $q(t)$ represent saturable gain and losses, respectively, and $\eta_{g}, \eta_{q}$ are the linewidth enhancement factors corresponding to self-phase modulation. The constants $g_{0}$ and $q_{0}$ stand for unsaturated gain and absorption. The constants $\gamma_{g}$ and $\gamma_{q}$ are the carrier density relaxation rates in the gain and absorbing sections; $E_{g}$ and $E_{q}$ are the saturation energies in these sections; the ratio $s=E_{g} / E_{q}$ is important for laser dynamics. Finally, $T$ stands for the cold cavity round-trip time, and $\sqrt{\kappa}$ is the linear non-resonant attenuation factor per pass. The parameter $g_{0}$ is proportional to the pump current, which is the physical control parameter.

Assume $(g(t), q(t), a(t))^{\top} \in \mathbb{R} \oplus \mathbb{R} \oplus \mathbb{C} \simeq \mathbb{R}^{4}=: \mathscr{V}$ and equip $\mathscr{V}$ with the natural $S^{1}$-representation (trivial on $(g, q)$-components and complex multiplication on the $a$-component). Clearly, system (3.1) is $S^{1}$-equivariant. In what follows, assuming the value $\alpha:=g_{o}$ to be the bifurcation parameter, we will show how Proposition 2.1 (resp. Theorem 2.1) can be used to study bifurcations of relative equilibria (resp. relative periodic solutions) for the network of identical oscillators (3.1) coupled in a $S_{5}$-symmetric fashion.

3.2. $S_{5}$-configuration of identical semiconductor lasers. Let $\mathfrak{f}: \mathbb{R} \times C([-T, 0] ; \mathscr{V}) \rightarrow \mathscr{V}$ be the map induced by the right-hand side of system (3.1). Put $V:=\mathscr{V}^{5}$ and define the map $f_{o}: \mathbb{R} \oplus C([-T, 0] ; V) \rightarrow$ $V$ by

$$
f_{o}\left(\alpha, x_{t}\right)=\left(\mathfrak{f}\left(\alpha, x_{t}^{1}\right), \mathfrak{f}\left(\alpha, x_{t}^{2}\right), \ldots, \mathfrak{f}\left(\alpha, x_{t}^{5}\right)\right)^{\top}
$$

where $x=\left(x^{1}, x^{2}, \ldots, x^{5}\right)^{\top} \in V$. Take the linear operator $C: \mathscr{V} \rightarrow \mathscr{V}$ with the matrix

$$
C:=\left[\begin{array}{ccc}
0 & 0 & 0 \\
0 & 0 & 0 \\
0 & 0 & e^{i \psi}
\end{array}\right]
$$

and let $\mathscr{C}: V \rightarrow V$ be given by the block matrix

$$
\mathscr{C}:=\left[\begin{array}{lllll}
0 & C & C & C & C \\
C & 0 & C & C & C \\
C & C & 0 & C & C \\
C & C & C & 0 & C \\
C & C & C & C & 0
\end{array}\right] .
$$

We are interested in solutions to the system

$$
\dot{x}=f\left(\alpha, \eta, x, x_{t}\right):=f_{o}\left(\alpha, x_{t}\right)+\eta \mathscr{C} x, \quad x \in V, \alpha, \eta \in \mathbb{R},
$$

where $\eta$ stands for the strength of coupling.

The group $S_{5}$ acts naturally on $V$ by permutation of the coordinates of the vector $x \in V$. This action suggests the orthogonal $S_{5} \times S^{1}$-representation on $V$ given by

$$
\left(h, e^{i \tau}\right) x=\left(e^{i \tau} x^{h(1)}, e^{i \tau} x^{h(2)}, \ldots, e^{i \tau} x^{h(5)}\right)^{\top}, \quad e^{i \tau} \in S^{1}, h \in S_{5},
$$


where $x=\left(x^{1}, x^{2}, \ldots, x^{5}\right)^{\top} \in V$ and $e^{i \tau}$ acts on $x^{i} \in \mathscr{V} \simeq \mathbb{R} \oplus \mathbb{R} \oplus \mathbb{C}$ trivially on the first two components and by complex multiplication on the $\mathbb{C}$-component $(i=1,2 \ldots, 5)$. Obviously, system (3.5) satisfies condition (A0).

Remark 3.1. Recall, if $\mathbf{S}(\bar{x})$ is a relative equilibrium for system (3.5), then symmetries of $\mathbf{S}(\bar{x})$ are completely determined by a (twisted) isotropy subgroup $\mathscr{G}_{\bar{x}}$ with respect to the $\mathscr{G}:=S_{5} \times S^{1}$-action.

Observe that $x_{o}(\alpha):=\left(\frac{\alpha}{\gamma_{g}}, \frac{q_{0}}{\gamma_{q}}, 0\right) \in \mathscr{V}$ is an equilibrium of system (3.1) for any $\alpha$, hence

$$
\mathscr{O}(\alpha):=\left(x_{o}(\alpha), x_{o}(\alpha), \ldots, x_{o}(\alpha)\right) \in V
$$

is an equilibrium of system (3.5) for any $\alpha$. Also (cf. (2.8)),

$$
D_{\mathbf{x}} \mathfrak{f}\left(\alpha, x_{o}(\alpha)\right)_{\mid \mathscr{V}}=\left[\begin{array}{ccc}
-\gamma_{g} & 0 & 0 \\
0 & -\gamma_{q} & 0 \\
0 & 0 & \left(\sqrt{\kappa} \exp \left[\frac{\left(1-i \eta_{g}\right) \frac{\alpha}{\gamma_{g}}-\left(1-i \eta_{q}\right) \frac{q_{0}}{\gamma_{q}}}{2}\right]-1\right) \gamma
\end{array}\right]
$$

\subsection{Bifurcation of symmetric relative equilibria.}

3.3.1. Isotypical decomposition and maximal twisted orbit types. To apply Proposition 2.1 for studying relative equilibria bifurcating from the equilibrium $\mathscr{O}(\alpha)$, observe that $V$ admits the isotypical $S_{5}$ decomposition:

$$
V=W_{0} \oplus W_{1},
$$

where

$$
W_{0}=\left\{\left(x^{1}, \ldots, x^{5}\right) \in V: x^{1}=\ldots=x^{5}\right\}
$$

is modeled on the one-dimensional trivial $S_{5}$-representation $\mathscr{V}_{0}$, while

$$
W_{1}=\left\{\left(x^{1}, \ldots, x^{5}\right) \in V: x^{1}+\ldots+x^{5}=0\right\}
$$

is modeled on the standard four-dimensional irreducible representation $\mathscr{V}_{4}$ (sometimes called augmentation submodule (cf. [20])). Clearly, $\operatorname{dim} W_{0}=4$ and $\operatorname{dim} W_{1}=16$. Observe also (see (2.13)) that decomposition (3.8) can be refined to the $S_{5} \times S^{1}$-decomposition

$$
W_{j}=V_{j, 0} \oplus V_{j, 1}, \quad j=0,1,
$$

where $V_{j, 0}\left(\right.$ resp. $\left.V_{j, 1}\right)$ corresponds to the trivial (resp. non-trivial) $S^{1}$-action. Clearly, $\operatorname{dim} V_{0,0}=$ $\operatorname{dim} V_{0,1}=2$ while $\operatorname{dim} V_{1,0}=\operatorname{dim} V_{1,1}=8$. Also, $V_{1,0}$ is reducible of multiplicity two (essentially modeled on the augmented module), while $V_{1,1}$ is irreducible.

The list of maximal twisted types in $V_{j, 1}, j=0,1$, is:

for $V_{0,1}: \quad\left(S_{5} \times\{1\}\right) \simeq\left(S_{5}\right)$;

for $V_{1,1}: \quad\left(D_{6}\right),\left(S_{4}\right),\left(D_{6}^{d}\right),\left(D_{4}^{d}\right),\left(\mathbb{Z}_{4}^{t}\right),\left(\mathbb{Z}_{5}^{t}\right),\left(\mathbb{Z}_{6}^{c}\right)$.

We refer to Subsection A.1 of the Appendix A for explicit description of all these subgroups, see also Remark 3.1. 
3.3.2. Equivariant spectral reduction and condition (A1). The linearization

$$
D_{\mathbf{x}} f_{o}(\alpha, \mathbf{x}): \mathbb{R} \times C_{-r}(V)_{\mid V} \rightarrow V
$$

of system (3.5) at $\mathscr{O}(\alpha)$ respects isotypical decomposition (3.11) (see also (3.8)). Then, setting

$$
A:=\left(\sqrt{\kappa} \exp \left[\frac{\left(1-i \eta_{g}\right) \frac{\alpha}{\gamma_{g}}-\left(1-i \eta_{q}\right) \frac{q_{0}}{\gamma_{q}}}{2}\right]-1\right) \gamma
$$

(cf. (3.7)), and combining (3.9)-(3.10) with (3.2)-(3.5), one obtains the following formulas for the restrictions $A_{j, k}:=D_{\mathbf{x}} f_{o}(\alpha, \mathbf{x}): \mathbb{R} \times C_{-r}(V)_{\mid V_{j, k}}, j, k=0,1$, to the isotypical components:

$$
A_{j, k}= \begin{cases}{\left[\begin{array}{cc}
-\gamma_{g} & 0 \\
0 & -\gamma_{q}
\end{array}\right]} & \text { if } j=0, k=0 ; \\
A+4 \eta e^{i \psi} & \text { if } j=0, k=1 ; \\
{\left[\begin{array}{cc}
-\gamma_{g} \mathrm{Id}_{4} & 0 \\
0 & -\gamma_{q} \mathrm{Id}_{4}
\end{array}\right]} & \text { if } j=1, k=0 ; \\
\left(A-\eta e^{i \psi}\right) \otimes \mathrm{Id}_{4} & \text { if } j=1, k=1,\end{cases}
$$

where $A+4 \eta e^{i \psi}$ and $A-\eta e^{i \psi}$ are considered as real $2 \times 2$-matrices, $\otimes$ stands for the Kronecker product of matrices, and $\mathrm{Id}_{4}$ denotes the identical $4 \times 4$-matrix. Since the action of $S^{1}$ on $(g, q)$-components of (3.1) is trivial, it follows from (3.13) that $\operatorname{det}\left(D_{\mathbf{x}} f_{o}(\alpha, \mathscr{O}(\alpha))_{\mid V^{1}}\right)=\left(\gamma_{g} \gamma_{q}\right)^{5} \neq 0$, hence (see (2.10)), $\mathscr{P}_{0}(\alpha, 0, \mathscr{O}(\alpha)) \neq 0$ so that system (3.5) satisfies condition (A1).

3.3.3. Characteristic quasi-polynomial and condition (A2). Next, let us consider the characteristic quasipolynomial $\mathscr{P}_{*}(\alpha, \lambda, \mathscr{O}(\alpha))$ (see (2.10)). Put

$$
P:=\lambda+\gamma-\gamma \sqrt{\kappa} \exp \left[\left(\frac{\alpha}{2 \gamma_{g}}-\frac{q_{0}}{2 \gamma_{q}}\right)+i\left(\frac{\eta_{q} q_{0}}{2 \gamma_{q}}-\frac{\eta_{g} \alpha}{2 \gamma_{g}}\right)\right] e^{-\lambda T} .
$$

Then, the restriction of the characteristic quasi-polynomial to $V_{j, 1}$ reads

$$
\mathscr{P}_{j, 1}(\alpha, \lambda, \mathscr{O}(\alpha))= \begin{cases}P+4 \eta e^{i \psi} & \text { if } j=0 \\ \left(P-\eta e^{i \psi}\right)^{4} & \text { if } j=1\end{cases}
$$

so that

$$
\mathscr{P}_{*}(\alpha, \lambda, \mathscr{O}(\alpha))=\mathscr{P}_{0,1}(\alpha, \lambda, \mathscr{O}(\alpha)) \cdot \mathscr{P}_{1,1}(\alpha, \lambda, \mathscr{O}(\alpha))
$$

To study condition (A2), we simplify the notations as follows (cf. (3.14)):

$$
x(\alpha):=\frac{\alpha}{2 \gamma_{g}}-\frac{q_{o}}{2 \gamma_{q}}, \quad y(\alpha):=\frac{\eta_{q} q_{o}}{2 \gamma_{q}}-\frac{\eta_{g} \alpha}{2 \gamma_{g}},
$$

and

$$
a_{j}+i b_{j}:= \begin{cases}-4 \eta e^{i \psi} & \text { if } j=0 \\ \eta e^{i \psi} & \text { if } j=1 .\end{cases}
$$

Let us identify the values of $\alpha$ for which $\mathscr{O}(\alpha)$ is a center, i.e. we are looking for those values of $\alpha$ for which there exists $w>0$ such that $\mathscr{P}_{*}(\alpha, i w, \mathscr{O}(\alpha))=0$. Equivalently (cf. (3.17)-(3.18)),

$$
i w=-\gamma+\gamma \sqrt{\kappa} \exp (x(\alpha)+i(y(\alpha)-w T))+a_{j}+i b_{j}, \quad j=0,1 .
$$


This complex equation can be reduced to the real equation

$$
\tan (y(\alpha)-w(\alpha) T)=\frac{w(\alpha)-b_{j}}{\gamma-a_{j}}, \quad j=0,1,
$$

with

$$
w(\alpha):=\gamma \sqrt{\kappa} e^{x(\alpha)} \sqrt{1-\frac{\left(\gamma-a_{j}\right)^{2}}{\gamma^{2} \kappa e^{2 x(\alpha)}}}+b_{j} .
$$

Clearly, (3.19) has infinitely many solutions $\alpha$ together with the corresponding limit frequencies $w(\alpha)$. Combining this with the same argument as in the proof of Proposition 4.2 in [4], one obtains the following

Lemma 3.1. Denote by $\lambda(\alpha)=r(\alpha)+i \omega(\alpha)$ the characteristic roots of (3.16) (see also (3.14)-(3.15)). Assume that $\alpha=\alpha_{o}^{j}$ is a root of (3.19), (3.20) for $j=0$ (resp. $\left.j=1\right)$, and

$$
\gamma>4 \eta \cos (\psi) \quad \text { and } \quad \omega\left(\alpha_{o}^{j}\right)>4 \eta \sin (\psi)
$$

(resp.

$$
\left.\gamma>-\eta \cos (\psi) \quad \text { and } \quad \omega\left(\alpha_{o}^{j}\right)>-\eta \sin (\psi)\right) .
$$

Then, $r^{\prime}\left(\alpha_{o}^{j}\right)>0$ (in particular, the center $\mathscr{O}\left(\alpha_{o}^{j}\right)$ is isolated and condition (iii) from Proposition 2.1 is satisfied).

3.3.4. Result. Combining Proposition 2.1 with the results presented in Subsubsections 3.3.1-3.3.3, one obtains the following result.

Proposition 3.1. Under the notations and assumptions of Lemma 3.1 (see also Subsection A.1 for the notations for subgroups), the following continuous branches of relative equilibria bifurcate from the equilibrium $\left(\alpha_{o}^{j}, \mathscr{O}\left(\alpha_{o}^{j}\right)\right)$ of equation (3.5):

for $j=0$, a branch with symmetry $\left(S_{5}\right)$;

for $j=1$, ten branches with symmetry $\left(D_{6}\right)$, five branches with symmetry $\left(S_{4}\right)$, ten branches with symmetry $\left(D_{6}^{d}\right)$, fifteen branches with symmetry $\left(D_{4}^{d}\right)$, thirty branches with symmetry $\left(\mathbb{Z}_{4}^{t_{1}}\right)$, twenty four branches with symmetry $\left(\mathbb{Z}_{5}^{t_{1}}\right)$, and twenty branches with symmetry $\left(\mathbb{Z}_{6}^{t_{2}}\right)$.

Recall that $\eta$ stands for the coupling strength. In particular, conditions (3.21) and (3.22) are satisfied for any relatively weak coupling.

Remark 3.2. Proposition 3.1 is illustrated in Table 1. We use the same set of parameters as in [4], i.e. $\eta=2, \eta_{g}=1, \eta_{q}=1, \gamma_{g}=10^{-2}, \gamma_{q}=1, \gamma=15, \kappa=\sqrt{0.2}, q_{0}=2, E_{g}=1, E_{q}=0.1, T=2.5$. Note that for these parameters, conditions (3.21) and (3.22) are fulfilled for all $(\alpha, \omega(\alpha))$ satisfying equations (3.19), (3.20). The equilibrium $\mathscr{O}(\alpha)$ is stable for $\alpha<0.036$. In Table 1 , we localize Hopf bifurcation points along the horizontal direction, and specify isotypical components $V_{j, 1}$ along the vertical direction. In each cell, we indicate the number of unstable roots of the corresponding characteristic polynomial $\mathscr{P}_{j, 1}$ defined by (3.15). One can easily see a change of stability as $\alpha$ increases. An entry of the table is circled to indicate a "jump" in the number of unstable roots and hence a Hopf bifurcation point. In particular, Proposition 3.1 guarantees Hopf bifurcations of branches of relative equilibria as follows:

(i) with symmetry $\left(S_{5}\right)$ for $\alpha \approx 0.036018, \alpha \approx 0.0361215, \alpha \approx 0.0364415$;

(ii) with symmetries $\left(D_{6}\right),\left(D_{6}^{d}\right),\left(S_{4}\right),\left(D_{4}^{d}\right),\left(\mathbb{Z}_{5}^{t_{1}}\right),\left(\mathbb{Z}_{4}^{t_{1}}\right)$ and $\left(\mathbb{Z}_{6}^{t_{2}}\right)$ for $\alpha \approx 0.0361515, \alpha \approx 0.0362365$, $\alpha \approx 0.0365865$, 
to mention a few (see Proposition 3.1 for the number of branches of each type and Subsection A.1 for the notations for subgroups).

TABLE 1. Number of unstable eigenvalues in each isotypical component for the equilibrium $\mathscr{O}(\alpha)$

\begin{tabular}{|c|c|c|c|c|c|c|c|c|c|}
\cline { 2 - 11 } \multicolumn{1}{c|}{} & \multicolumn{7}{|c|}{ Intervals for values of parameter $\alpha \cdot 10^{2}$} \\
\cline { 2 - 11 } \multicolumn{1}{c|}{} & {$[3.6000,3.6016]$} & {$[3.6020,3.6119]$} & {$[3.6124,3.6149]$} & {$[3.6154,3.6234]$} & {$[3.6239,3.6439]$} & {$[3.6444,3.6584]$} & {$[3.6589,3.6744]$} & {$[3.6749,3.6829]$} & {$[3.6834,3.6959]$} \\
\hline$V_{0,1}$ & 0 & 2 & 4 & 4 & 4 & 6 & 6 & 8 & 8 \\
\hline$V_{1,1}$ & 0 & 0 & 0 & 8 & 16 & 16 & 24 & 24 & 32 \\
\hline$\bigoplus_{j=0}^{1} V_{j, 1}$ & 0 & 2 & 4 & 12 & 20 & 22 & 30 & 32 & 40 \\
\hline
\end{tabular}

\subsection{Bifurcation of relative periodic solutions.}

3.4.1. Application of Theorem 2.1 to the laser system. In this subsection, we show how Theorem 2.1 can be applied to classify symmetries of relative periodic solutions, which bifurcate from branches of relative equilibria of system (3.5) (see Remark 3.2 and Appendix A). Further, an infinite number of Hopf bifurcations of relative periodic solutions occurs along each branch of relative equilibria. To be specific, we consider a few successive Hopf bifurcations at the beginning of each branch of our choice. In contrast to the application of Proposition 2.1 to studying bifurcation of relative equilibria (in which case, all the necessary symbolic computations were explicitly presented), we have to resort to numerical computations for verifying conditions (A3), (A5) and (ii), (iii) of Theorem 2.1.

Based on the numerical evidence, Theorem 2.1 allows us to predict the following bifurcations of branches of relative periodic solutions.

(a) The following branches of relative periodic solutions bifurcate from $\left(S_{5}\right)$-symmetric branch of relative equilibria:

(i) with symmetry $\left(S_{5}\right)$ for $\alpha \approx 0.0744915, \alpha \approx 0.612203$;

(ii) with symmetries $\left(D_{6}\right),\left(S_{4}\right),\left(D_{6}^{d}\right),\left(D_{4}^{d}\right),\left(\mathbb{Z}_{4}^{t_{1}}\right),\left(\mathbb{Z}_{5}^{t}\right),\left(\mathbb{Z}_{6}^{t_{2}}\right)$ for $\alpha \approx 0.054159, \alpha \approx 0.190194$, $\alpha \approx 0.2253355$.

(b) The following branches of relative periodic solutions bifurcate from $\left(\mathbb{Z}_{5}^{t_{1}}\right)$-symmetric branch of relative equilibria:

(i) with symmetry $\left(\mathbb{Z}_{5}^{t_{1}}\right)$ for $\alpha \approx 0.072873$;

(ii) with symmetries $\left(\mathbb{Z}_{5}^{t_{1}, t_{1}}\right)$ for $\alpha \approx 0.0367485, \alpha \approx 0.072873$.

(c) The following branches of relative periodic solutions bifurcate from $\left(D_{6}^{d}\right)$-symmetric branch of relative equilibria:

(i) with symmetries $\left(\mathbb{Z}_{6}^{t_{3}, t_{1}}\right),\left(D_{2}^{d, \boldsymbol{d}}\right)$ and $\left(D_{2}^{d, \hat{\boldsymbol{d}}}\right)$ for $\alpha \approx 0.0362275, \alpha \approx 0.036575, \alpha \approx 0.036825$, $\alpha \approx 0.037475, \alpha \approx 0.037825$

(ii) with symmetry $\left(D_{6}^{d, d}\right)$ for $\alpha \approx 0.0363745, \alpha \approx 0.036675, \alpha \approx 0.037125, \alpha \approx 0.037175$, $\alpha \approx 0.037525, \alpha \approx 0.037625$.

Remark 3.3. Note that branches of relative periodic solutions with symmetries $\left(\mathbb{Z}_{6}^{t_{3}, t_{2}}\right),\left(D_{2}^{d}\right)$ and $\left(D_{2}^{d, z}\right)$ do not bifurcate from $\left(D_{6}^{d}\right)$ symmetric relative equilibrium because $S^{1}$ acts trivially in isotypical component $U_{2}$ (see case (c) in Subsubsection 3.4.2). 
(d) The following branches of relative periodic solutions bifurcate from $\left(S_{4}\right)$-symmetric branch of relative equilibria:

(i) with symmetry $\left(S_{4}\right)$ for $\alpha \approx 0.0365895$;

(ii) with symmetries $\left(\mathbb{Z}_{4}^{t_{2}}\right),\left(D_{4}^{d}\right),\left(D_{2}^{d}\right)$ and $\left(\mathbb{Z}_{3}^{t_{1}}\right)$ for $\alpha \approx 0.0362445, \alpha \approx 0.0366295$;

Symmetries of bifurcating branches split into different classes related to different isotypical components (see Subsubsection 3.4.2 for details). In the remaining cases, we can predict the following symmetric properties of bifurcating branches of relative periodic solutions without numerical simulations (very similar to cases (a)-(d)).

(e) The following branches of relative periodic solutions bifurcate from $\left(D_{6}\right)$-symmetric branch of relative equilibria:

(i) with symmetry $\left(D_{6}\right)$;

(ii) with symmetries $\left(\mathbb{Z}_{6}^{\boldsymbol{t}_{2}}\right),\left(D_{2}\right)$ and $\left(D_{2}^{z}\right)$;

(iii) with symmetry $\left(D_{6}^{d}\right)$.

(f) The following branches of relative periodic solutions bifurcate from $\left(\mathbb{Z}_{6}^{t_{2}}\right)$-symmetric branch of relative equilibria:

(i) with symmetry $\left(\mathbb{Z}_{6}^{t_{2}}\right)$;

(ii) with symmetry $\left(\mathbb{Z}_{6}^{t_{2}, t_{1}}\right)$;

(iii) with symmetry $\left(\mathbb{Z}_{6}^{t_{2}, t_{2}}\right)$;

(iv) with symmetry $\left(\mathbb{Z}_{6}^{t_{2}, t_{3}}\right)$.

(g) The following branches of relative periodic solutions bifurcate from $\left(D_{4}^{d}\right)$-symmetric branch of relative equilibria:

(i) with symmetry $\left(D_{4}^{d}\right)$;

(ii) with symmetries $\left(\mathbb{Z}_{4}^{d, t_{1}}\right),\left(D_{2}^{d}\right)$ and $\left(D_{2}^{\hat{d}}\right)$;

(iii) with symmetry $\left(D_{4}^{d, d}\right)$.

(h) The following branches of relative periodic solutions bifurcate from $\left(\mathbb{Z}_{4}^{t_{1}}\right)$-symmetric branch of relative equilibria:

(i) with symmetry $\left(\mathbb{Z}_{4}^{t_{1}}\right)$;

(ii) with symmetry $\left(\mathbb{Z}_{4}^{t_{1}, t_{1}}\right)$;

(iii) with symmetry $\left(\mathbb{Z}_{4}^{t_{1}, t_{2}}\right)$.

\subsection{2. $\mathscr{G}_{\bar{x}}$-isotypical decomposition of $V^{c}$ and maximal twisted orbit types.}

In this subsubsection, we discuss isotypical decompositions, maximal twisted orbit types and restrictions of the matrix $\mathscr{C}$ to isotypical components relevant to cases (a)-(h) in Subsubsection 3.4.1.

(a) We consider the case $\mathscr{H}:=S_{5} \times\{1\}$. The complexification $\mathscr{V}^{c}$ of the space $\mathscr{V}:=\mathbb{R}^{2} \oplus \mathbb{C}=\mathbb{R}^{2} \oplus$ $(\mathbb{R} \oplus \mathbb{R})$ can be represented as

$$
\mathscr{V}^{c}=\mathbb{C}^{2} \oplus(\mathbb{C} \oplus \mathbb{C})=\mathbb{C}^{4}
$$

thus $V^{c}=\left(\mathscr{V}^{c}\right)^{5}=\left(\mathbb{C}^{4}\right)^{5}$. First, we consider the subgroup $\mathbb{Z}_{5} \simeq \mathbb{Z}_{5} \times\{1\}$ and describe the $\mathbb{Z}_{5}$-isotypical decomposition of $V^{c}$. Namely, we have $V^{c}=U_{0} \oplus U_{1} \oplus U_{2}$, where $U_{0}=\left\{(z, z, z, z, z)^{\top}: z \in \mathbb{C}^{4}\right\}, U_{j}=$ $U_{j}^{+} \oplus U_{j}^{-}, j=1,2$,

$$
U_{j}^{ \pm}=\left\{\left(z, \xi^{ \pm j} z, \xi^{ \pm 2 j} z, \xi^{ \pm 3 j} z, \xi^{ \pm 4 j} z\right)^{\top}: z \in \mathbb{C}^{4}\right\} \quad j=1,2, \xi=e^{\frac{2 \pi}{5}}
$$


One can easily notice that the coupling matrix $\mathscr{C}: V^{c} \rightarrow V^{c}$ given by (3.4) preserves the $\mathbb{Z}_{5}$-isotypical components. By inspection,

$$
\begin{aligned}
\left.\mathscr{C}\right|_{U_{0}}(z, z, z, z, z)^{\top} & =4(C z, C z, C z, C z, C z)^{\top}, \quad z \in \mathbb{C}^{4} \\
\left.\mathscr{C}\right|_{U_{j}^{ \pm}}\left(z, \xi^{ \pm j} z, \xi^{ \pm 2 j} z, \xi^{ \pm 3 j} z, \xi^{ \pm 4 j} z\right)^{\top} & =-\left(C z, \xi^{ \pm j} C z, \xi^{ \pm 2 j} C z, \xi^{ \pm 3 j} C z, \xi^{ \pm 4 j} z C\right)^{\top}, \quad z \in \mathbb{C}^{4} .
\end{aligned}
$$

Notice that en element $\zeta \in \mathbf{S}$ acts on $\left(z_{1}, z_{2}, z_{3}, z_{4}\right)^{\top} \in \mathbb{C}^{4}$ by

$$
\zeta\left(z_{1}, z_{2}, z_{3}, z_{4}\right)^{\top}=\left(z_{1}, z_{2}, \zeta z_{3}, \zeta z_{4}\right)^{\top} \text {. }
$$

Using the above $\mathbb{Z}_{5}$-isotypical decomposition, we obtain for $\mathscr{H}:=S_{5} \times\{1\}$ the following $S_{5}$-isotypical decomposition of $V^{c}$ :

$$
V^{c}=U_{0} \oplus U_{1}
$$

where $U_{1}=U_{1}^{+} \oplus U_{1}^{-} \oplus U_{2}^{+} \oplus U_{2}^{-}$. Moreover

$$
\left.\mathscr{C}\right|_{U_{0}}=\left[\begin{array}{cccc}
0 & 0 & 0 & 0 \\
0 & 0 & 0 & 0 \\
0 & 0 & 4 \cos \psi & -4 \sin \psi \\
0 & 0 & 4 \sin \psi & 4 \cos \psi
\end{array}\right],\left.\quad \mathscr{C}\right|_{U_{j}^{ \pm}}=\left[\begin{array}{cccc}
0 & 0 & 0 & 0 \\
0 & 0 & 0 & 0 \\
0 & 0 & -\cos \psi & \sin \psi \\
0 & 0 & -\sin \psi & -\cos \psi
\end{array}\right], j=1,2 .
$$

Hence, for the $\mathscr{H}:=S_{5}$-representation $V^{c}$, we have the following list of maximal twisted types in the isotypical components:

(i) for $U_{0,1}: \quad\left(S_{5}\right)$;

(ii) for $U_{1,1}: \quad\left(D_{6}\right),\left(S_{4}\right),\left(D_{6}^{d}\right),\left(D_{4}^{d}\right),\left(\mathbb{Z}_{4}^{t_{1}}\right),\left(\mathbb{Z}_{5}^{t_{1}}\right),\left(\mathbb{Z}_{6}^{t_{2}}\right)$.

(b) We consider now the group $\mathscr{H}:=\mathbb{Z}_{5}^{t_{1}}:=\left\{(1,1),(\xi, \xi),\left(\xi^{2}, \xi^{2}\right),\left(\xi^{3}, \xi^{3}\right),\left(\xi^{4}, \xi^{4}\right)\right\}$. Put $\mathscr{W}:=$ $\left\{\left(z^{1}, z^{2}, z^{3}, z^{4}, z^{5}\right)^{\top}: z^{k}=\left(z_{1}, z_{2}, 0,0\right)^{\top} \in \mathbb{C}^{4}\right\}$ and $\mathscr{V}:=\left\{\left(z^{1}, z^{2}, z^{3}, z^{4}, z^{5}\right)^{\top}: z^{k}=\left(0,0, z_{1}, z_{2}\right)^{\top} \in \mathbb{C}^{4}\right\}$, and define

$$
W_{0}:=\left\{(z, z, z, z, z)^{\top}: z \in \mathscr{W}\right\}, \quad V_{0}:=\left\{(z, z, z, z, z)^{\top}: z \in \mathscr{V}\right\}
$$

and

$$
\begin{aligned}
W_{j}^{ \pm} & =\left\{\left(z, \xi^{ \pm j} z, \xi^{ \pm 2 j} z, \xi^{ \pm 3 j} z, \xi^{ \pm 4 j} z\right)^{\top}: z \in \mathscr{W}\right\}, \\
V_{j}^{ \pm} & =\left\{\left(z, \xi^{ \pm j} z, \xi^{ \pm 2 j} z, \xi^{ \pm 3 j} z, \xi^{ \pm 4 j} z\right)^{\top}: z \in \mathscr{V}\right\}, \quad j=1,2, \xi=e^{\frac{2 \pi i}{5}} .
\end{aligned}
$$

Then, one can easily verify that we have the following $\mathscr{H}$-isotypical decomposition of $V^{c}$ :

$$
V^{c}=U_{0} \oplus U_{1} \oplus U_{2}
$$

where $U_{0}=W_{0} \oplus V_{1}^{-}, U_{1}=V_{0} \oplus V_{2}^{-}$and $U_{2}=V_{1}^{+} \oplus V_{2}^{+}$. 
Moreover $\left.\mathscr{C}\right|_{W_{0}}=\left.\mathscr{C}\right|_{W_{j}^{ \pm}}=0$,

$$
\begin{gathered}
\left.\mathscr{C}\right|_{V_{1}^{+}}=\left[\begin{array}{cc}
-\cos \psi & \sin \psi \\
-\sin \psi & -\cos \psi
\end{array}\right],\left.\quad \mathscr{C}\right|_{V_{0}}=\left[\begin{array}{cc}
4 \cos \psi & -4 \sin \psi \\
4 \sin \psi & 4 \cos \psi
\end{array}\right] \\
\left.\mathscr{C}\right|_{V_{2}^{+}}=\left[\begin{array}{cc}
-\cos \psi & \sin \psi \\
-\sin \psi & -\cos \psi
\end{array}\right],\left.\quad \mathscr{C}\right|_{V_{j}^{-}}=\left[\begin{array}{cc}
-\cos \psi & \sin \psi \\
-\sin \psi & -\cos \psi
\end{array}\right]
\end{gathered}
$$

where $j=1,2$. Hence, for the $\mathscr{H}:=\mathbb{Z}_{5}^{t_{1}}$-representation $V^{c}$, we have the following list of maximal twisted types in the isotypical components:

(i) for $U_{0,1}: \quad\left(\mathbb{Z}_{5}^{t_{1}}\right)$;

(ii) for $U_{1,1}$ and $U_{2,1}: \quad\left(\mathbb{Z}_{5}^{t_{1}, t_{1}}\right)$.

(c) Next, we consider $\mathscr{H}:=D_{6}^{d}$. Again, put $\mathscr{W}:=\left\{\left(z^{1}, z^{2}, z^{3}, z^{4}, z^{5}\right)^{\top}: z^{k}=\left(z_{1}, z_{2}, 0,0\right)^{\top} \in \mathbb{C}^{4}\right\}$ and $\mathscr{V}:=\left\{\left(z^{1}, z^{2}, z^{3}, z^{4}, z^{5}\right)^{\top}: z^{k}=\left(0,0, z_{1}, z_{2}\right)^{\top} \in \mathbb{C}^{4}\right\}$ and define

$$
\begin{aligned}
W_{0} & =\left\{\left(z^{1}, z^{1}, z^{2}, z^{2}, z^{1}\right)^{\top}: z^{1}, z^{2} \in \mathscr{W}\right\} \\
W_{2} & =W_{2}^{+} \oplus W_{2}^{-} \\
W_{2}^{ \pm} & :=\left\{\left(z, \rho^{ \pm 2} z, 0,0, \rho^{ \pm 4} z\right)^{\top}: z \in \mathscr{W}\right\}, \quad \rho:=e^{\frac{2 \pi i}{6}} \\
W_{3} & =\left\{(0,0, z,-z, 0)^{\top}: z \in \mathscr{W}\right\}
\end{aligned}
$$

and

$$
\begin{aligned}
V_{0} & =\left\{\left(z^{1}, z^{1}, z^{2}, z^{2}, z^{1}\right)^{\top}: z_{1}, z^{2} \in \mathscr{V}\right\} \\
V_{2} & =V_{2}^{+} \oplus V_{2}^{-} \\
V_{2}^{ \pm} & :=\left\{\left(z, \rho^{ \pm 2} z, 0,0, \rho^{ \pm 4} z\right)^{\top}: z \in \mathscr{V}\right\}, \quad \rho:=e^{\frac{2 \pi i}{6}} \\
V_{3} & =\left\{(0,0, z,-z, 0)^{\top}: z \in \mathscr{V}\right\}
\end{aligned}
$$

Then by inspection, we have the following $\mathscr{H}$-isotypical decomposition of $V^{c}$ :

$$
V^{c}=U_{0} \oplus U_{1} \oplus U_{2} \oplus U_{3}
$$

where

$$
\begin{aligned}
& U_{0}=W_{0} \oplus V_{3} \\
& U_{1}=V_{2} \\
& U_{2}=W_{2} \\
& U_{3}=W_{3} \oplus V_{0}
\end{aligned}
$$


The restrictions of $\mathscr{C}$ to components $U_{j}$ are given by

$$
\begin{gathered}
\left.\mathscr{C}\right|_{U_{0}}=\mathbf{0}_{6 \times 6},\left.\quad \mathscr{C}\right|_{U_{1}}=\left[\begin{array}{ccccc}
-\cos \psi & \sin \psi & 0 & 0 \\
-\sin \psi & -\cos \psi & 0 & 0 \\
0 & 0 & -\cos \psi & \sin \psi \\
& 0 & 0 & -\sin \psi & -\cos \psi
\end{array}\right] \\
\left.\mathscr{C}\right|_{U_{2}}=\mathbf{0}_{4 \times 4},\left.\quad \mathscr{C}\right|_{U_{3}}=\left[\begin{array}{cccccc}
0 & 0 & 0 & 0 & 0 & 0 \\
0 & 0 & 0 & 0 & 0 & 0 \\
0 & 0 & 4 \cos \psi & -4 \sin \psi & 0 & 0 \\
0 & 0 & 4 \sin \psi & 4 \cos \psi & 0 & 0 \\
0 & 0 & 0 & 0 & -\cos \psi & \sin \psi \\
0 & 0 & 0 & 0 & -\sin \psi & -\cos \psi
\end{array}\right] .
\end{gathered}
$$

Hence, for the $\mathscr{H}:=D_{6}^{d}$-representation $V^{c}$, we have the following list of maximal twisted types in the isotypical components:

(i) for $U_{0,1}: \quad\left(D_{6}^{d}\right)$;

(ii) for $U_{1,1}: \quad\left(\mathbb{Z}_{6}^{t_{3}, \boldsymbol{t}_{1}}\right),\left(D_{2}^{d, \boldsymbol{d}}\right)$ and $\left(D_{2}^{d, \hat{\boldsymbol{d}}}\right)$;

(iii) for $U_{2,1}: \quad\left(\mathbb{Z}_{6}^{t_{3}, t_{2}}\right),\left(D_{2}^{d}\right)$ and $\left(D_{2}^{d, z}\right)$;

(iv) for $U_{3,1}: \quad\left(D_{6}^{d, \boldsymbol{d}}\right)$.

(d) In the next case, we consider $\mathscr{H}:=S_{4}$. One can easily verify that the $\mathscr{H}$-isotypical decomposition of $V^{c}$ is given by

$$
V^{c}=U_{0} \oplus U_{4},
$$

where the component $U_{4}$ is modeled on the irreducible $S_{4}$-representation which is equivalent to the augmentation submodule of $S_{4}$. These components are exactly:

$$
\begin{aligned}
& U_{0}=\left\{\left(z^{1}, z^{2}, z^{2}, z^{2}, z^{2}\right)^{\top}: z^{1}, z^{2} \in \mathbb{C}^{4}\right\} \\
& U_{4}=\left\{\left(0, z^{1}, z^{2}, z^{3}, z^{4}\right)^{\top}: z^{1}+z^{2}+z^{3}+z^{4}=0\right\} .
\end{aligned}
$$


Put $U_{0}^{1}:=\left\{(z, z, z, z, z)^{\top}: z \in \mathbb{C}^{4}\right\}$ and $U_{0}^{2}:=\left\{(-4 z, z, z, z, z)^{\top}: z \in \mathbb{C}^{4}\right\}$. Then, we can represent the restriction of $\mathscr{C}$ to each component as follows:

$$
\begin{gathered}
\left.\mathscr{C}\right|_{U_{0}^{1}}=\left[\begin{array}{cccc}
0 & 0 & 0 & 0 \\
0 & 0 & 0 & 0 \\
0 & 0 & 4 \cos \psi & -4 \sin \psi \\
0 & 0 & 4 \sin \psi & 4 \cos \psi
\end{array}\right],\left.\quad \mathscr{C}\right|_{U_{0}^{2}}=\left[\begin{array}{cccc}
0 & 0 & 0 & 0 \\
0 & 0 & 0 & 0 \\
0 & 0 & -\cos \psi & \sin \psi \\
0 & 0 & -\sin \psi & -\cos \psi
\end{array}\right] \\
\left.\mathscr{C}\right|_{U_{4}}=\left[\begin{array}{cccc}
0 & 0 & 0 & 0 \\
0 & 0 & 0 & 0 \\
0 & 0 & -\cos \psi & \sin \psi \\
0 & 0 & -\sin \psi & -\cos \psi
\end{array}\right]
\end{gathered}
$$

Hence, for the $\mathscr{H}:=S_{4}$-representation $V^{c}$, we have the following list of maximal twisted types in the isotypical components:

(i) for $U_{0,1}: \quad\left(S_{4}\right)$;

(ii) for $U_{4,1}: \quad\left(\mathbb{Z}_{4}^{t_{2}}\right),\left(D_{4}^{d}\right),\left(D_{2}^{d}\right)$ and $\left(\mathbb{Z}_{3}^{t_{1}}\right)$.

Observe that, although the isotypical component $U_{4,1}$ contains a maximal twisted orbit type $\left(D_{3}\right)$, we cannot guarantee the existence of a branch with this symmetry since $U_{0,1}$ contains $\left(S_{4}\right)$ as a maximal twisted orbit type.

(e) Now we consider the isotropy group $\mathscr{H}:=D_{6}$, where $D_{6}=\mathbb{Z}_{6} \cup \mathbb{Z}_{6} \kappa$, and

$$
\begin{aligned}
\mathbb{Z}_{6} & =\{(1),(125)(34),(152),(34),(125),(152)(34)\} \\
\mathbb{Z}_{6} \kappa & =\{(12),(25)(34),(15),(12)(34),(25),(15)(34)\}
\end{aligned}
$$

On can easily verify that we have the following $\mathscr{H}$-isotypical decomposition of $V^{c}: V^{c}=U_{0} \oplus U_{2} \oplus U_{3}$, where

$$
\begin{aligned}
U_{0} & =\left\{\left(z^{1}, z^{1}, z^{2}, z^{2}, z^{1}\right)^{\top}: z^{1}, z^{2} \in \mathbb{C}^{4}\right\} \\
U_{2} & =U_{2}^{+} \oplus U_{2}^{-} \\
U_{2}^{ \pm} & :=\left\{\left(z, \rho^{ \pm 2} z, 0,0, \rho^{ \pm 4} z\right)^{\top}: z \in \mathbb{C}^{4}\right\}, \quad \rho:=e^{\frac{2 \pi i}{6}} \\
U_{3} & =\left\{(0,0, z,-z, 0)^{\top}: z \in \mathbb{C}^{4}\right\} .
\end{aligned}
$$


Put $U_{0}^{1}:=\left\{(z, z, z, z, z)^{\top}: z \in \mathbb{C}^{4}\right\}$ and $U_{0}^{2}:=\left\{\left(z, z,-\frac{3}{2} z,-\frac{3}{2} z, z\right)^{\top}: z \in \mathbb{C}^{4}\right\}$, so that $U_{0}=U_{0}^{1} \oplus U_{0}^{2}$. Then,

$$
\begin{gathered}
\left.\mathscr{C}\right|_{U_{0}^{1}}=\left[\begin{array}{cccc}
0 & 0 & 0 & 0 \\
0 & 0 & 0 & 0 \\
0 & 0 & 4 \cos \psi & -4 \sin \psi \\
0 & 0 & 4 \sin \psi & 4 \cos \psi
\end{array}\right],\left.\quad \mathscr{C}\right|_{U_{0}^{2}}=\left[\begin{array}{cccc}
0 & 0 & 0 & 0 \\
0 & 0 & 0 & 0 \\
0 & 0 & -\cos \psi & \sin \psi \\
0 & 0 & -\sin \psi & -\cos \psi
\end{array}\right] \\
\left.\mathscr{C}\right|_{U_{2}^{ \pm}}=\left[\begin{array}{cccc}
0 & 0 & 0 & 0 \\
0 & 0 & 0 & 0 \\
0 & 0 & -\cos \psi & \sin \psi \\
0 & 0 & -\sin \psi & -\cos \psi
\end{array}\right],\left.\mathscr{C}\right|_{U_{3}}=\left[\begin{array}{cccc}
0 & 0 & 0 & 0 \\
0 & 0 & 0 & 0 \\
0 & 0 & 0 & 0 \\
0 & 0 & 0 & 0
\end{array}\right] .
\end{gathered}
$$

Hence, for the $\mathscr{H}:=D_{6}$-representation $V^{c}$, we have the following list of maximal twisted types in the isotypical components:

(i) for $U_{0,1}:\left(D_{6}\right)$;

(ii) for $U_{2,1}: \quad\left(\mathbb{Z}_{6}^{t_{2}}\right),\left(D_{2}\right)$ and $\left(D_{2}^{z}\right)$;

(iii) for $U_{3,1}: \quad\left(D_{6}^{d}\right)$.

(f) Let us consider the group $\mathscr{H}:=\mathbb{Z}_{6}^{t_{2}}$. Then, using the decompositions in (d), we obtain the following $\mathscr{H}$-isotypical decomposition of $V^{c}$ :

$$
V^{c}=U_{0} \oplus U_{1} \oplus U_{2} \oplus U_{3}
$$

where

$$
\begin{aligned}
& U_{0}=W_{0} \oplus V_{2}^{+}, \\
& U_{1}=V_{3}, \\
& U_{2}=W_{2} \oplus V_{2}^{-} \oplus V_{0}, \\
& U_{3}=W_{3}
\end{aligned}
$$

(we follow the same notation as in (c)). Again, the restrictions of $\mathscr{C}$ to components $U_{j}$ can be easily described using the relations established in (e). Hence, for the $\mathscr{H}:=\mathbb{Z}_{6}^{t_{2}}$-representation $V^{c}$, we have the following list of maximal twisted types in the isotypical components:

(i) for $U_{0,1}: \quad\left(\mathbb{Z}_{6}^{t_{2}}\right)$;

(ii) for $U_{1,1}: \quad\left(\mathbb{Z}_{6}^{t_{2}, t_{1}}\right)$;

(iii) for $U_{2,1}: \quad\left(\mathbb{Z}_{6}^{t_{2}, t_{2}}\right)$;

(iv) for $U_{3,1}: \quad\left(\mathbb{Z}_{6}^{t_{2}, t_{3}}\right)$. 
(g) We consider now the group $\mathscr{H}:=D_{4}^{d}$, where

$$
\begin{aligned}
D_{4}^{d}=\{ & ((1), 1),((2345),-1),((24)(35), 1),((2543),-1),((24), 1),((25)(34),-1),((35), 1), \\
& ((23)(45),-1)\},
\end{aligned}
$$

and put $\mathscr{W}:=\left\{\left(z^{1}, z^{2}, z^{3}, z^{4}, z^{5}\right)^{\top}: z^{k}=\left(z_{1}, z_{2}, 0,0\right)^{\top} \in \mathbb{C}^{4}\right\}$ and $\mathscr{V}:=\left\{\left(z^{1}, z^{2}, z^{3}, z^{4}, z^{5}\right)^{\top}: z^{k}=\left(0,0, z_{1}, z_{2}\right)^{\top}\right.$ $\left.\in \mathbb{C}^{4}\right\}$. We have the following $\mathbb{Z}_{4}$-invariant components in $V^{c}$ :

$$
\begin{array}{rlrl}
W_{0}^{1} & :=\left\{(0, z, z, z, z)^{\top}: z \in \mathscr{W}\right\}, & V_{0}^{1}:=\left\{(z, z, z, z, z)^{\top}: z \in \mathscr{V}\right\}, \\
W_{0}^{2}:=\left\{(z, 0,0,0,0)^{\top}: z \in \mathscr{W}\right\}, & V_{0}^{2}:=\left\{(-4 z, z, z, z, z)^{\top}: z \in \mathscr{V}\right\}, \\
W_{1}^{ \pm}:=\left\{(0, z, \pm i z,-z, \mp i z)^{\top}: z \in \mathscr{W}\right\}, & V_{1}^{ \pm}:=\left\{(0, z, \pm i z,-z, \mp i z)^{\top}: z \in \mathscr{V}\right\} \\
W_{2}:=\left\{(0, z,-z, z,-z)^{\top}: z \in \mathscr{W}\right\}, & V_{2}:=\left\{(0, z,-z, z,-z)^{\top}: z \in \mathscr{V}\right\} .
\end{array}
$$

Then, the $D_{4}^{d}$-isotypical decomposition of $V^{c}$ is

$$
V^{c}=U_{0} \oplus U_{1} \oplus U_{2}
$$

where

$$
U_{0}=W_{0}^{1} \oplus W_{0}^{2} \oplus V_{2}, \quad U_{1}=W_{1}^{+} \oplus W_{1}^{-} \oplus V_{1}^{+} \oplus V_{1}^{-}, \quad U_{2}=V_{0}^{1} \oplus V_{0}^{2} \oplus W_{2} .
$$

Then, we can represent the restriction of $\mathscr{C}$ to $U$ by

$$
\begin{gathered}
\left.\mathscr{C}\right|_{V_{2}}=\left[\begin{array}{cc}
-\cos \psi & \sin \psi \\
-\sin \psi & -\cos \psi
\end{array}\right],\left.\quad \mathscr{C}\right|_{V_{1}^{ \pm}}=\left[\begin{array}{cc}
-\cos \psi & \sin \psi \\
-\sin \psi & -\cos \psi
\end{array}\right] \\
\left.\mathscr{C}\right|_{V_{0}^{1}}=\left[\begin{array}{cc}
4 \cos \psi & -4 \sin \psi \\
4 \sin \psi & 4 \cos \psi
\end{array}\right],\left.\quad \mathscr{C}\right|_{V_{0}^{2}}=\left[\begin{array}{cc}
-\cos \psi & \sin \psi \\
-\sin \psi & -\cos \psi
\end{array}\right] \\
\left.\mathscr{C}\right|_{W_{0}^{1}}=\left.\mathscr{C}\right|_{W_{0}^{2}}=\left.\mathscr{C}\right|_{W_{1}^{ \pm}}=\left.\mathscr{C}\right|_{W_{2}} \equiv 0
\end{gathered}
$$

Hence, for the $\mathscr{H}:=D_{4}^{d}$-representation $V^{c}$, we have the following list of maximal twisted types in the isotypical components:

(i) for $U_{0,1}: \quad\left(D_{4}^{d}\right)$;

(ii) for $U_{1,1}: \quad\left(\mathbb{Z}_{4}^{d, t_{1}}\right)$ and $\left(D_{2}^{d}\right)=\left(D_{2}^{\hat{d}}\right)$;

(iii) for $U_{2,1}: \quad\left(D_{4}^{d, d}\right)$.

(h) We consider now the group $\mathscr{H}:=\mathbb{Z}_{4}^{t_{1}}$, where

$$
\mathbb{Z}_{4}^{t_{1}}=\{((1), 1),((2345), i),((24)(35),-1),((2543),-i)\} .
$$

Consider again the following $\mathbb{Z}_{4}$-invariant components in $V^{c}$ :

$$
\begin{array}{rlrl}
W_{0}^{1} & :=\left\{(0, z, z, z, z)^{\top}: z \in \mathscr{W}\right\}, & V_{0}^{1}:=\left\{(z, z, z, z, z)^{\top}: z \in \mathscr{V}\right\}, \\
W_{0}^{2}:=\left\{(z, 0,0,0,0)^{\top}: z \in \mathscr{W}\right\}, & V_{0}^{2}:=\left\{(-4 z, z, z, z, z)^{\top}: z \in \mathscr{V}\right\}, \\
W_{1}^{ \pm}:=\left\{(0, z, \pm i z,-z, \mp i z)^{\top}: z \in \mathscr{W}\right\}, & V_{1}^{ \pm}:=\left\{(0, z, \pm i z,-z, \mp i z)^{\top}: z \in \mathscr{V}\right\} \\
W_{2}:=\left\{(0, z,-z, z,-z)^{\top}: z \in \mathscr{W}\right\}, & V_{2}:=\left\{(0, z,-z, z,-z)^{\top}: z \in \mathscr{V}\right\} .
\end{array}
$$


Then, the $\mathbb{Z}_{4}^{t}$-isotypical decomposition of $V^{c}$ is

$$
V^{c}=U_{0} \oplus U_{1} \oplus U_{2}
$$

where

$$
U_{0}=W_{0}^{1} \oplus W_{0}^{2} \oplus V_{1}^{+}, \quad U_{1}=W_{1}^{+} \oplus W_{1}^{-} \oplus V_{2} \oplus V_{0}^{1} \oplus V_{0}^{2}, \quad U_{2}=W_{2} \oplus V_{1}^{-} .
$$

Then, we can represent the restriction of $\mathscr{C}$ to each component as follows:

$$
\begin{gathered}
\left.\mathscr{C}\right|_{V_{2}}=\left[\begin{array}{cc}
-\cos \psi & \sin \psi \\
-\sin \psi & -\cos \psi
\end{array}\right],\left.\quad \mathscr{C}\right|_{V_{1}^{ \pm}}=\left[\begin{array}{cc}
-\cos \psi & \sin \psi \\
-\sin \psi & -\cos \psi
\end{array}\right], \\
\left.\mathscr{C}\right|_{V_{0}^{1}}=\left[\begin{array}{cc}
4 \cos \psi & -4 \sin \psi \\
4 \sin \psi & 4 \cos \psi
\end{array}\right],\left.\quad \mathscr{C}\right|_{V_{0}^{2}}=\left[\begin{array}{cc}
-\cos \psi & \sin \psi \\
-\sin \psi & -\cos \psi
\end{array}\right], \\
\left.\mathscr{C}\right|_{W_{0}^{1}}=\left.\mathscr{C}\right|_{W_{0}^{2}}=\left.\mathscr{C}\right|_{W_{1}^{ \pm}}=\left.\mathscr{C}\right|_{W_{2}} \equiv 0
\end{gathered}
$$

Hence, for the $\mathscr{H}:=\mathbb{Z}_{6}^{t_{2}}$-representation $V^{c}$, we have the following list of maximal twisted types in the isotypical components:

(i) for $U_{0,1}: \quad\left(\mathbb{Z}_{4}^{t_{1}}\right)$;

(ii) for $U_{1,1}: \quad\left(\mathbb{Z}_{4}^{t_{1}, t_{1}}\right)$;

(iii) for $U_{2,1}: \quad\left(\mathbb{Z}_{4}^{t_{1}, t_{2}}\right)$.

3.4.3. Linearization on a relative equilibrium and characteristic quasi-polynomials. For any $\bar{x}^{o}=(g, q, a) \in$ $\mathbb{R} \oplus \mathbb{R} \oplus \mathbb{C} \simeq \mathscr{V}$, one has (cf. (2.5)-(2.6) and (3.1)):

$$
\widetilde{\mathfrak{f}}\left(\alpha, i \omega, \bar{x}^{o}\right)=\left[\begin{array}{c}
\alpha-\gamma_{g} g-\frac{1}{E_{g}} e^{-q}\left(e^{g}-1\right)|a|^{2} \\
q_{0}-\gamma_{q} q-\frac{1}{E_{q}}\left(1-e^{-q}\right)|a|^{2} \\
-\gamma a+\gamma \sqrt{\kappa} \exp \left[\frac{\left(1-i \eta_{g}\right) g-\left(1-i \eta_{q}\right) q}{2}\right] a e^{-i \omega T}
\end{array}\right] .
$$

Take $\lambda \in \mathbb{C}$. Combining (3.26) with (2.22), (2.23) and (2.25) allows us to define a "linearization operator" $\mathscr{R}_{\alpha}^{\mathscr{V}}(\lambda): \mathscr{V}^{c} \rightarrow \mathscr{V}^{c}$ by

$$
\mathscr{R}_{\alpha}^{\mathscr{V}}(\lambda):=\left[\begin{array}{ccc}
-\gamma_{g}-\frac{1}{E_{g}} e^{-q} e^{g}|a|^{2} & \frac{1}{E_{g}} e^{-q}\left(e^{g}-1\right)|a|^{2} & -\frac{2}{E_{g}} e^{-q}\left(e^{g}-1\right) a \\
0 & -\gamma_{q}-\frac{1}{E_{q}} e^{-q}|a|^{2} & -\frac{2}{E_{q}}\left(1-e^{-q}\right) a \\
B_{31}(\lambda) & B_{32}(\lambda) & B_{33}(\lambda)
\end{array}\right],
$$

where

$$
\begin{aligned}
& B_{31}(\lambda)=\frac{\gamma \sqrt{\kappa}\left(1-i \eta_{g}\right)}{2} \exp \left[\frac{\left(1-i \eta_{g}\right) g-\left(1-i \eta_{q}\right) q}{2}\right] a e^{-i \omega T-\lambda T} \\
& B_{32}(\lambda)=-\frac{\gamma \sqrt{\kappa}\left(1-i \eta_{q}\right)}{2} \exp \left[\frac{\left(1-i \eta_{g}\right) g-\left(1-i \eta_{q}\right) q}{2}\right] a e^{-i \omega T-\lambda T} \\
& B_{33}(\lambda)=-\gamma+\gamma \sqrt{\kappa} \exp \left[\frac{\left(1-i \eta_{g}\right) g-\left(1-i \eta_{q}\right) q}{2}\right] a e^{-i \omega T-\lambda T} .
\end{aligned}
$$

For any $\bar{x}=\left(\bar{x}^{1}, \ldots, \bar{x}^{5}\right) \in V$, put

$$
\widetilde{f}_{o}(\alpha, i \omega, \bar{x}):=\left(\widetilde{\mathfrak{f}}\left(\alpha, i \omega, \bar{x}^{1}\right), \ldots, \tilde{\mathfrak{f}}\left(\alpha, i \omega, \bar{x}^{5}\right)\right)^{\top} .
$$


For a given $\alpha, \mathbf{S}(\bar{x}(\alpha))$ is a relative equilibrium for system (3.5) corresponding to the frequency $\omega(\alpha)$ if and only if

$$
\Phi(\alpha, \omega(\alpha), \bar{x}(\alpha)):=\widetilde{f}_{o}(\alpha, i \omega(\alpha), \bar{x}(\alpha))+\eta \mathscr{C} \bar{x}(\alpha)-\omega(\alpha) J \bar{x}(\alpha)=0
$$

(cf. (2.12)). Assume that $\mathbf{S}(\bar{x}(\alpha))$ is a relative equilibrium with $\mathscr{H}:=\mathscr{G}_{\bar{x}(\alpha)}$ of the form listed in Remark 3.2 (see Condition (A4)). Take $\mathscr{R}_{\alpha}(\lambda)$ determined by (3.29) and (2.22)-(2.23) and consider decomposition (3.24). Then (cf. (3.27) and (3.29)), one has:

$$
\left.\mathscr{R}_{\alpha}(\lambda)\right|_{\mathfrak{U}}= \begin{cases}\mathscr{R}_{\alpha}^{\mathscr{V}}(\lambda)+\eta \mathscr{C}_{0} & \text { if } \mathfrak{U}=U_{0} ; \\ \mathscr{R}_{\alpha}^{\mathscr{V}}(\lambda)+\eta \mathscr{C}_{1} & \text { if } \mathfrak{U}=U_{1},\end{cases}
$$

where $\mathscr{C}_{0}$ and $\mathscr{C}_{1}$ are determined by formulas (3.25). Combining (3.30) with (2.24) and (2.28), one can define the characteristic quasi-polynomials $\overline{\mathscr{P}}_{j, 1}(\alpha, \lambda)$ and study the Hopf bifurcation of relative periodic solutions for different values of $\mathscr{H}=S_{5}, \mathbb{Z}_{5}^{t_{1}}, D_{6}^{d}, S_{4}, D_{6}, D_{4}^{d}, \mathbb{Z}_{6}^{t_{2}}, \mathbb{Z}_{4}^{t_{1}}$.

3.4.4. Condition (A3). Our argument is close to the one used in [4]. Suppose that for some $\bar{\alpha}$ and $\bar{w} \in \mathbb{R}$, equation (3.5) has a relative equilibrium $\mathbf{S}(\bar{x})$, and assume, for simplicity, that $\bar{x}=\left(x, \omega_{5} x, \omega_{5}^{2} x, \omega_{5}^{3} x, \omega_{5}^{4} x\right)^{\top}$, $\omega_{5}:=e^{\frac{2 \pi i}{5}}, x=(g, q, a) \in \mathscr{V}$ (in which case $\left.\mathscr{G}_{\bar{x}}=\mathbb{Z}_{5}^{t_{1}}\right)$, or $\bar{x}=(0,0,0, x,-x)^{\top}$ (in which case $\mathscr{G}_{\bar{x}}=D_{6}^{d}$ ); see Appendix B (the general case can be treated in a similar way).

Without loss of generality, assume that $a \in \mathbb{C}$ is real. Take decomposition (3.8) and let us describe the restriction of matrix (2.19) to $\mathbb{R} \times W_{j}$. For $j=0,1$, define the operator $\mathscr{B}_{j}=\mathscr{B}_{j}(\bar{\alpha}, \bar{w}, \bar{x}): \mathscr{V} \rightarrow \mathscr{V}$ by

$$
\mathscr{B}_{j}:= \begin{cases}\mathscr{R}_{\bar{\alpha}}^{\mathscr{V}}(0)+4 \eta C-\bar{w} J^{\mathscr{V}} & \text { if } j=0 ; \\ \mathscr{R}_{\bar{\alpha}}^{\mathscr{V}}(0)-\eta C-\bar{w} J^{\mathscr{V}} & \text { if } j=1 .\end{cases}
$$

Here $\mathscr{R}_{\bar{\alpha}}^{\mathscr{V}}(0)$ is considered as a real linear operator in $\mathscr{V} \simeq \mathbb{R}^{2} \oplus \mathbb{C}$ (cf. (3.27)) and $J^{\mathscr{V}}: \mathscr{V} \rightarrow \mathscr{V}$ is given by $J^{\mathscr{V}}(\tilde{g}, \tilde{q}, \tilde{a})^{\top}=(0,0, i \tilde{a})^{\top}$; see also (3.3). Define a vector $\mathscr{B}=\mathscr{B}(\bar{\alpha}, \bar{w}, \bar{x}) \in \mathscr{V} \simeq \mathbb{R}^{2} \oplus \mathbb{C}$ by

$$
\mathscr{B}:=\left\{0,0,-i T \gamma \sqrt{\kappa} \exp \left[\frac{\left(1-i \eta_{g}\right) g-\left(1-i \eta_{q}\right) q}{2}\right] a e^{-i \omega T}-i a\right\}^{\top}
$$

Put $\mathfrak{B}:=\left[\mathscr{B} \mid \mathscr{B}_{j}\right]$. Then (see (2.19), (3.26), (3.31) and (3.32)),

$$
\left[D_{w} \Phi(\bar{\alpha}, \bar{w}, \bar{x}) \mid D_{x} \Phi(\bar{\alpha}, \bar{w}, \bar{x})\right]_{\mathbb{R} \times W_{j}}=\left\{\begin{array}{llll}
\mathfrak{B} & & & \text { if } j=0, \\
{\left[\begin{array}{cccc}
\mathfrak{B} & 0 & 0 & 0 \\
0 & \mathfrak{B} & 0 & 0 \\
0 & 0 & \mathfrak{B} & 0 \\
0 & 0 & 0 & \mathfrak{B}
\end{array}\right] \quad \text { if } j=1 .}
\end{array}\right.
$$

It follows from (3.33) that condition (A3) is satisfied if $\operatorname{rank}(\mathfrak{B})=4$. Note that $(0,0, i)^{\top} \in \mathbb{R}^{2} \oplus \mathbb{C}$ is an eigenvector of $\mathscr{B}_{j}$ corresponding to the zero eigenvalue. Denote by $\mathscr{E}$ the direct sum of generalized eigenspaces corresponding to non-zero eigenvalues of $\mathscr{B}_{j}$. Clearly, $\operatorname{rank}(\mathfrak{B})=4$ if

(a) $\operatorname{rank}\left(\mathscr{B}_{j}\right)=3$ (i.e., zero is a simple eigenvalue of $\mathscr{B}_{j}$ ), and

(b) $\mathfrak{B} e \notin \mathscr{E}$, where $e:=(1,0,0,0,0) \in \mathbb{R}^{5} \simeq \mathbb{R} \oplus \mathbb{R}^{2} \oplus \mathbb{C} \simeq \mathbb{R} \oplus \mathscr{V}$.

Remark 3.4. Condition (a) can be effectively expressed in terms of the derivative of the characteristic polynomial associated with $\mathscr{B}_{j}$. Condition (b) is satisfied if 
$(\mathrm{b})^{\prime} \operatorname{Im}\left[-i T \gamma \sqrt{\kappa} \exp \left[\frac{\left(1-i \eta_{g}\right) g-\left(1-i \eta_{q}\right) q}{2}\right] a e^{-i \omega T}\right]-a \neq 0$

(recall, $a \in \mathbb{R})$.

3.4.5. Isotypical crossing. In order to apply Theorem 2.1 to classify symmetries of relative periodic solutions bifurcating from relative equilibria $\mathbf{S}(\bar{x})$ with $\left(\mathscr{G}_{\bar{x}}\right)$ listed in Remark 3.2, it remains to analyze the isotypical crossing of the roots of characteristic quasi-polynomials $\overline{\mathscr{P}}_{j, 1}(\alpha, \lambda)$ (cf. (3.30), (2.24) and (2.28)), as $\alpha$ crosses some critical value $\alpha_{o}$. Numerical results illustrating isotypical crossing of characteristic roots through the imaginary axis are described in Table 2 for $\left(\mathscr{G}_{\bar{x}}\right)=S_{5}$, in Table 3 for $\left(\mathscr{G}_{\bar{x}}\right)=\mathbb{Z}_{5}^{t_{1}}$, in Table 4 for $\left(\mathscr{G}_{\bar{x}}\right)=S_{4}$, and in Table 5 for $\left(\mathscr{G}_{\bar{x}}\right)=D_{6}^{d}$. All parameters except $\alpha$ are the same as in Subsection 3.3. In these tables, we follow the same agreement as in Table 1. In particular, an entry in a given cell indicates the number of unstable roots for the characteristic quasi-polynomial $\overline{\mathscr{P}}_{j, 1}(\alpha, \lambda)$ associated with the isotypical component $U_{j, 1}$ (shown in the left column) for the corresponding interval of $\alpha$-values (shown in the upper row). The results presented in Subsubsection 3.4.1 follow from these tables.

TABLE 2. Number of unstable eigenvalues for each isotypical component along the branch of the relative equilibrium with $\left(S_{5}\right)$ symmetry

\begin{tabular}{|c|c|c|c|c|c|c|}
\cline { 2 - 7 } \multicolumn{1}{c|}{} & \multicolumn{7}{c|}{ Intervals for values of parameter $\alpha \cdot 10^{2}$} \\
\cline { 2 - 7 } & {$[3.6020,5.1788]$} & {$[5.6530,7.1953]$} & {$[7.7030,18.7684]$} & {$[19.2704,22.2825]$} & {$[22.7846,60.9689]$} & {$[61.4717,91.6423]$} \\
\hline$U_{0,1}$ & 0 & 0 & 2 & 2 & 2 & 4 \\
\hline$U_{1,1}$ & 0 & 4 & 4 & 12 & 20 & 20 \\
\hline$\bigoplus_{j=0}^{1} U_{j, 1}$ & 0 & 4 & 6 & 14 & 22 & 24 \\
\hline
\end{tabular}

TABLE 3. Number of unstable eigenvalues for each isotypical component along the branch of the relative equilibrium with $\left(\mathbb{Z}_{5}^{t_{1}}\right)$ symmetry

\begin{tabular}{|c|c|c|c|c|c|c|c|c|c|c|c|c|}
\hline & \multicolumn{12}{|c|}{ Intervals for values of parameter $\alpha \cdot 10^{2}$} \\
\hline & {$[3.6154,3.6215]$} & {$[3.6240,3.6349]$} & {$[3.6400,3.6550]$} & {$[3.6600,3.6650]$} & {$[3.6700,3.6800]$} & {$[3.6850,3.7100]$} & {$[3.7150,3.7150]$} & {$[3.7200,3.7450]$} & {$[3.7500,3.7500]$} & {$[3.7550,3.7600]$} & {$[3.7650,3.7800]$} & {$[3.7850,3.8450]$} \\
\hline$U_{0,1}$ & 0 & 0 & 0 & 0 & 0 & 0 & 0 & 0 & 0 & 0 & 0 & 0 \\
\hline$U_{1,1}$ & 4 & 8 & 8 & (12) & 12 & (16) & 16 & 16 & (20) & 20 & 20 & (24) \\
\hline$U_{2,1}$ & 5 & 5 & (7) & 7 & (9) & 9 & (11) & 13 & 13 & 15 & (17) & 17 \\
\hline$\bigoplus_{j=0}^{2} U_{j, 1}$ & 9 & 13 & 15 & 19 & 21 & 25 & 27 & 29 & 33 & 35 & 37 & 41 \\
\hline
\end{tabular}


TABLE 4. Number of unstable eigenvalues for each isotypical component along the branch of the relative equilibrium with $\left(S_{4}\right)$ symmetry

\begin{tabular}{|c|c|c|c|c|c|c|}
\cline { 2 - 7 } \multicolumn{1}{c|}{} & \multicolumn{6}{c|}{ Intervals for values of parameter $\alpha \cdot 10^{2}$} \\
\cline { 2 - 7 } & {$[3.6154,3.6242]$} & {$[3.6247,3.6587]$} & {$[3.6592,3.6627]$} & {$[3.6632,3.6902]$} & {$[3.6907,3.7058]$} & {$[3.7063,3.7118]$} \\
\hline$U_{0,1}$ & 4 & 4 & 6 & 6 & 6 & 8 \\
\hline$U_{1,1}$ & 6 & 12 & 12 & 18 & 24 & 24 \\
\hline$\bigoplus_{j=0}^{1} U_{j, 1}$ & 10 & 16 & 18 & 24 & 30 & 32 \\
\hline
\end{tabular}

TABLE 5. Number of unstable eigenvalues for each isotypical component along the branch of the relative equilibrium with $\left(D_{6}^{d}\right)$ symmetry

\begin{tabular}{|c|c|c|c|c|c|c|c|c|c|c|c|c|}
\hline & \multicolumn{12}{|c|}{ Intervals for values of parameter $\alpha \cdot 10^{2}$} \\
\hline & {$[3.6154,3.6215]$} & {$[3.6240,3.6349]$} & {$[3.6400,3.6550]$} & {$[3.6600,3.6650]$} & {$[3.6700,3.6800]$} & {$[3.6850,3.7100]$} & {$[3.7150,3.7150]$} & {$[3.7200,3.7450]$} & {$[3.7500,3.7500]$} & {$[3.7550,3.7600]$} & {$[3.7650,3.7800]$} & {$[3.7850,3.8450]$} \\
\hline$U_{0,1}$ & 0 & 0 & 0 & 0 & 0 & 0 & 0 & 0 & 0 & 0 & 0 & 0 \\
\hline$U_{1,1}$ & 4 & (8) & 8 & (12) & 12 & (16) & 16 & 16 & (20) & 20 & 20 & (24) \\
\hline$U_{3,1}$ & 5 & 5 & (7) & 7 & (9) & 9 & (11) & (13) & 13 & (15) & (17) & 17 \\
\hline$\bigoplus_{j=0,1,3} U_{j, 1}$ & 9 & 13 & 15 & 19 & 21 & 25 & 27 & 29 & 33 & 35 & 37 & 41 \\
\hline
\end{tabular}

\section{Appendix A. Maximal Twisted Subgroups and Equivariant Jargon}

If $\mathfrak{W}$ is a $G$-representation, then for any function $x: S^{1} \rightarrow \mathfrak{W}$, the spatio-temporal symmetry of $x$ is a group $\mathscr{H}<G \times S^{1}$ such that $g \cdot x(t-\theta)=x(t)$ for any $t \in \mathbb{R} / 2 \pi \mathbb{Z} \simeq S^{1}$ and any $\left(g, e^{i \theta}\right) \in \mathscr{H}$. If $x$ is non-constant, then $\mathscr{H}$ has the structure of a graph of a homomorphism $\varphi: H \rightarrow S^{1}$, where $H$ is some subgroup of $G$. To emphasize this nature of the group $\mathscr{H}$, the following notation is commonly used:

$$
\mathscr{H}:=H^{\varphi}=\{(h, \varphi(h)): h \in H\} .
$$

The group $H^{\varphi}$ is called a twisted symmetry group with twisting homomorpism $\varphi$. In particular, denote $\mathscr{H}:=H$ if $\varphi$ is trivial.

Relative periodic solutions of our interest have symmetry groups $\mathscr{K}$ which are subgroups of $\Gamma \times S^{1} \times$ $S^{1}$. Such subgroups $\mathscr{K}$ can be characterized by two twisting homomorphisms $\varphi: K \rightarrow S^{1}$ and $\psi: K^{\varphi} \rightarrow$ $S^{1}$ for some subgroup $K<\Gamma$, in which case, denote $\mathscr{K}:=K^{\varphi, \psi}$. In order to simplify our notations, we also adopt the following convention: given $\mathscr{K}=K^{\varphi, \psi}$, denote (a) $\mathscr{K}:=K^{\varphi}$ (resp. $\mathscr{K}:=K^{\boldsymbol{\psi}}$ ) if $\psi$ (resp. $\varphi$ ) is trivial and, (b) $\mathscr{K}:=K$ if both $\varphi$ and $\psi$ are trivial. In addition, we put $\omega_{k}:=\mathrm{e}^{\frac{2 \pi i}{k}}$. 
A.1. Notations used for selected twisted subgroups of $\mathscr{H}=S_{5} \times S^{1}$. The following symbols are used for selected twisted subgroups of $\mathscr{H}$ :

$$
\begin{aligned}
S_{5} & :=S_{5} \times\{1\}, \\
\mathbb{Z}_{5}^{t_{1}} & :=\left\langle\left((12345), \omega_{5}\right)\right\rangle, \\
D_{6} & :=D_{6} \times\{1\}, \\
D_{6}^{d} & :=\langle((12), 1),((123), 1),((45),-1)\rangle=\langle((123)(45),-1),((12), 1)\rangle, \\
\mathbb{Z}_{6}^{t_{2}} & :=\left\langle((45), 1),\left((123), \omega_{3}\right)\right\rangle=\left\langle\left((123)(45), \omega_{3}\right)\right\rangle, \\
S_{4} & :=S_{4} \times\{1\}, \\
D_{4}^{d} & :=\langle((24), 1),((35), 1),((2345),-1)\rangle=\langle((2345),-1),((24), 1)\rangle, \\
\mathbb{Z}_{4}^{t_{1}} & :=\left\langle\left((2345), \omega_{4}\right)\right\rangle .
\end{aligned}
$$

A.2. Notations used for selected twisted subgroups of $\mathscr{K}:=S_{5} \times\{1\} \times S^{1}$. The following symbols are used for selected twisted subgroups of $\mathscr{K}$ :

$$
\begin{aligned}
S_{5} & :=S_{5} \times\{1\} \times\{1\}, \\
D_{6} & :=D_{6} \times\{1\} \times\{1\}, \\
S_{4} & :=S_{4} \times\{1\} \times\{1\}, \\
D_{6}^{d} & :=\langle((12), 1,1),((125), 1,1),((34), 1,-1)\rangle=\langle(((125)(34), 1,-1),(12), 1,1)\rangle, \\
D_{4}^{d} & :=\langle((24), 1,1),((35), 1,1),((2345), 1,-1)\rangle=\langle((2345), 1,-1),((24), 1,1)\rangle, \\
\mathbb{Z}_{5}^{\boldsymbol{t}_{1}} & :=\left\langle\left((12345), 1, \omega_{5}\right)\right\rangle, \\
\mathbb{Z}_{4}^{\boldsymbol{t}_{1}} & :=\left\langle\left((2345), 1, \omega_{4}\right)\right\rangle, \\
\mathbb{Z}_{6}^{\boldsymbol{t}_{2}} & :=\left\langle((34), 1,1),\left((125), 1, \omega_{3}\right)\right\rangle=\left\langle\left((125)(34), 1, \omega_{3}\right)\right\rangle .
\end{aligned}
$$

A.3. Notations used for selected twisted subgroups of $\mathscr{K}:=\mathbb{Z}_{5}^{t_{l}} \times S^{1}$. In this case, the following symbols are used for selected twisted subgroups of $\mathscr{K}$ :

$$
\begin{aligned}
\mathbb{Z}_{5}^{t_{1}} & :=\mathbb{Z}_{5}^{t_{1}} \times\{1\}, \\
\mathbb{Z}_{5}^{t_{1}, t_{1}} & :=\left\langle\left((12345), \omega_{5}, \omega_{5}\right\rangle .\right.
\end{aligned}
$$

A.4. Notations used for selected twisted subgroups of $\mathscr{K}:=D_{6} \times S^{1}$. For this group, the following symbols are used for selected twisted subgroups of $\mathscr{K}$ :

$$
\begin{aligned}
& D_{6}:=D_{6} \times\{1\} \times\{1\}, \\
& \mathbb{Z}_{6}^{t_{2}}:=\left\langle((34), 1,1),\left((125), 1, \omega_{3}\right)\right\rangle=\left\langle\left((125)(34), 1, \omega_{3}\right\rangle,\right. \\
& D_{2}:=D_{2} \times\{1\} \times\{1\} \\
& D_{2}^{z}:=\langle((24)(35), 1,1),((24), 1,-1)\rangle, \\
& D_{6}^{d}:=\langle((125), 1,1),((12), 1,1),((34), 1,-1)\rangle=\langle((125)(34), 1,-1),((12), 1,1)\rangle .
\end{aligned}
$$


A.5. Notations used for selected twisted subgroups of $\mathscr{K}:=D_{6}^{d} \times S^{1}$. For this group, the following symbols are used for selected twisted subgroups of $\mathscr{K}$ :

$$
\begin{aligned}
D_{6}^{d} & :=D_{6}^{d} \times\{1\}, \\
\mathbb{Z}_{6}^{t_{3}, t_{1}} & :=\left\langle\left((125)(34),-1, \omega_{6}\right)\right\rangle, \\
D_{2}^{d, \boldsymbol{d}}: & :=\langle((12), 1,1),((12)(34),-1,-1)\rangle, \\
D_{2}^{d, \hat{\boldsymbol{d}}}: & =\langle((34),-1,1),((12)(34),-1,-1)\rangle, \\
\mathbb{Z}_{6}^{t_{3}, t_{2}} & :=\left\langle\left((125)(34),-1, \omega_{3}\right)\right\rangle, \\
D_{2}^{d} & :=D_{2}^{d} \times\{1\}, \\
D_{2}^{d, z}: & :=\langle((12)(34),-1,1),((12), 1,-1)\rangle, \\
D_{6}^{d, \boldsymbol{d}}: & :=\langle((12), 1,1),((125), 1,1),((34),-1,-1)\rangle=\langle((125)(34),-1,-1),((12), 1,1)\rangle .
\end{aligned}
$$

A.6. Notations used for selected twisted subgroups of $\mathscr{K}:=\mathbb{Z}_{6}^{t_{2}} \times S^{1}$. For this group, the following symbols are used for selected twisted subgroups of $\mathscr{K}$ :

$$
\begin{aligned}
\mathbb{Z}_{6}^{t_{2}} & :=\mathbb{Z}_{6}^{t_{2}} \times\{1\} \\
\mathbb{Z}_{6}^{t_{2}, t_{2}} & :=\left\langle((34), 1,1),\left((125), \omega_{3}, \omega_{3}\right)\right\rangle=\left\langle\left((125)(34), \omega_{3}, \omega_{3}\right)\right\rangle, \\
\mathbb{Z}_{6}^{t_{2}, t_{3}} & :=\left\langle((34), 1,-1),\left((125), \omega_{3}, 1\right)\right\rangle=\left\langle\left((125)(34), \omega_{3},-1\right)\right\rangle .
\end{aligned}
$$

A.7. Notations used for selected twisted subgroups of $\mathscr{K}:=S_{4} \times S^{1}$. For this group, the following symbols are used for selected twisted subgroups of $\mathscr{K}$ :

$$
\begin{aligned}
S_{4} & :=S_{4} \times\{1\} \times\{1\} \\
\mathbb{Z}_{4}^{t_{2}} & :=\langle((2345), 1,-1)\rangle \\
D_{4}^{d} & :=\langle((24), 1,1),((35), 1,1),((2345), 1,-1)\rangle=\langle((2345), 1,-1),((24), 1,1)\rangle, \\
D_{2}^{d} & :=\langle((24), 1,1),((24)(35), 1,-1)\rangle \\
\mathbb{Z}_{3}^{t_{1}} & :=\left\langle\left((234), 1, \omega_{3}\right)\right\rangle
\end{aligned}
$$

A.8. Notations used for selected twisted subgroups of $\mathscr{K}:=D_{4}^{d} \times S^{1}$. For this group, the following symbols are used for selected twisted subgroups of $\mathscr{K}$ :

$$
\begin{aligned}
D_{4}^{d} & :=D_{4}^{d} \times\{1\}, \\
\mathbb{Z}_{4}^{d, t_{1}} & :=\left\langle\left((2345),-1, \omega_{4}\right)\right\rangle, \\
D_{2}^{d} & :=\langle((24), 1,1),((35), 1,-1)\rangle \\
D_{2}^{\hat{d}} & :=\langle((24), 1,1),((35), 1,-1)\rangle \\
D_{4}^{d, d} & :=\langle((24), 1,1),((35), 1,1),((2345),-1,-1)\rangle=\langle((2345),-1,-1),((24), 1,1)\rangle .
\end{aligned}
$$


A.9. Notations used for selected twisted subgroups of $\mathscr{K}:=\mathbb{Z}_{4}^{t_{1}} \times S^{1}$. For this group, the following symbols are used for selected twisted subgroups of $\mathscr{K}$ :

$$
\begin{aligned}
\mathbb{Z}_{4}^{t_{1}} & :=\mathbb{Z}_{4}^{t_{1}} \times\{1\}, \\
\mathbb{Z}_{4}^{t_{1}, t_{1}} & :=\left\langle\left((2345), \omega_{4}, \omega_{4}\right)\right\rangle, \\
\mathbb{Z}_{4}^{t_{1}, z} & :=\left\langle\left((2345), \omega_{4},-1\right)\right\rangle .
\end{aligned}
$$

A.10. Equivariant Jargon. Let $G$ be a compact Lie group. Given a (closed) subgroups $H \subset G$, denote by $(H)$ the conjugacy class of $H$ in $G$. The set of all conjugacy classes of subgroups in $G$ is denoted by $\Phi(G)$. Clearly, $\Phi(G)$ admits a partial order defined by:

$$
(H) \leq(K) \quad \Leftrightarrow \quad \exists_{g \in G} g H g^{-1} \subset K .
$$

Let $X$ be a $G$-space and $x \in X$. We denote by $G_{x}:=\{g \in G: g x=x\}$ the isotropy (or stabilizer) of $x$, by $G(x):=\{g x: g \in G\} \simeq G / G_{x}$ the orbit of $x$. The conjugacy class $\left(G_{x}\right)$ will be called the orbit type of $x$. We will also adopt the following notation: $X^{H}:=\left\{x \in X: G_{x} \supset H\right\}$.

For two $G$-spaces $X$ and $Y$, a continuous map $f: X \rightarrow Y$ is said to be equivariant if $f(g x)=g f(x)$ for all $x \in X$ and $g \in G$. If the $G$-action on $Y$ is trivial, then $f$ is called invariant. Clearly, for any subgroup $H \subset G$ and equivariant map $f: X \rightarrow Y$, the map $f^{H}: X^{H} \rightarrow Y^{H}$, with $f^{H}:=\left.f\right|_{X^{H}}$, is well-defined. Finally, given two orthogonal $G$-representations $W$ and $V$ and an open bounded subset $\Omega \subset W$, an equivariant map $f: \Omega \rightarrow V$ is called $\Omega$-admissible if $f(x) \neq 0$ for all $x \in \partial \Omega$.

For further details of the equivariant jargon used in this paper, we refer to [9, 10, 27, 47]; for the representation theory background, see [11,47].

\section{Appendix B. EFFective Acting Group And Symmetry}

Let $V$ be an orthogonal representation of a compact Lie groop $G$ and let

$$
\dot{\mathbf{x}}=g(\mathbf{x}) \quad(\mathbf{x} \in V)
$$

be a $G$-equivariant system. Then, for any (closed) subgroup $H<G$, the subspace $V^{H}$ is flow invariant. In what follows, a restriction of (B.1) to $V^{H}$ will be called a reduced system. In general, a reduced system may have a very complicated form. In this section, based on our numerical computations, we are going to formulate a conjecture on reduced systems related to nonzero components of a relative equilibrium.

To simplify our exposition, consider a $\Gamma \times S^{1}$-symmetric linearly coupled system

$$
\dot{x_{i}}=f\left(x_{i}\right)+(\mathfrak{C x})_{i}, \quad i=1, \ldots n,
$$

where $\mathfrak{C}: \mathbb{R}^{m n} \rightarrow \mathbb{R}^{m n}$ is $\Gamma$-equivariant, $x_{i} \in \mathbb{R}^{m}$ and $\mathbf{x}=\left(x_{1}, \ldots, x_{n}\right)^{\top}$. Suppose there is a relative equilibrium $S(v)$, where $v=\left(x_{1}, \ldots, x_{n}\right)^{\top}$, satisfying

$$
x_{k} \equiv x_{k+1} \equiv \cdots \equiv x_{n}=0
$$

for some $k=1, \ldots, n$. Let $\mathfrak{H} \leq \Gamma \times S^{1}$ be the symmetry of $S(v)$ and

$$
\mathfrak{G}_{0}:=\left\{g \in \mathfrak{G}: g \mathbf{x}=\mathbf{x} \quad \forall \mathbf{x} \in \mathbb{R}^{m n} \text { such that } x_{1}=\cdots=x_{k-1}=0\right\} .
$$

Put $\mathfrak{H}_{0}:=\mathfrak{H} \cap \mathfrak{G}_{0}$. In such a case, we call $\mathfrak{G}_{0}$ the effective acting group and $\mathfrak{H}_{0}$ the effective symmetry of $S(v)$. 
Example B.1. Let $\Gamma=S_{5}$ and consider the system with $\Gamma \times S^{1}$-symmetric linear coupling:

$$
\dot{x}_{i}=f\left(x_{i}\right)+\alpha \sum_{1 \leq j \leq 5, j \neq i} x_{j}
$$

for $i=1, \ldots, 5$ (we assume that the $\Gamma \times S^{1}$-action is similar to the one considered in Subsection 3.2). Case 1: $\mathfrak{H}=\mathbb{Z}_{5}^{t_{1}}$. A relative equilibrium with $\mathfrak{H}$-symmetry takes the form $\mathbf{S}(v)$, where $v=\left(x, \omega_{5} x, \omega_{5}^{2} x, \omega_{5}^{3} x\right.$, $\left.\omega_{5}^{4} x\right)^{\top}$. Its effective acting group is $\mathfrak{G}_{0}=S_{5} \times S^{1}$ and effective symmetry is $\mathfrak{H}_{0}=\mathbb{Z}_{5}^{t_{1}}$. Also in such a case, (B.3) reduces to

$$
\begin{aligned}
\dot{x} & =f(x)+\alpha \sum_{j=1}^{4} \omega_{5}^{j} x \\
& =f(x)-\alpha x .
\end{aligned}
$$

Case 2: $\mathfrak{H}=D_{6}^{d}$. A relative equilibrium with $\mathfrak{H}$-symmetry takes the form $\mathbf{S}(v)$, where $v=(0,0,0, x,-x)^{\top}$. Its effective acting group is $\mathfrak{G}_{0}=S_{2} \times S^{1}$ and effective symmetry is $\mathfrak{H}_{0}=\mathbb{Z}_{2}^{t_{1}}\left(S_{2} \simeq \mathbb{Z}_{2}\right)$. Also, in such a case, (B.3) reduces to

$$
\dot{x}=f(x)-\alpha x .
$$

Case 3: $\mathfrak{H}=S_{4}$. A relative equilibrium with $\mathfrak{H}$-symmetry takes the form $\mathbf{S}(v)$, where $v=(x, x, x, x, y)^{\top}$. Its effective acting group is $\mathfrak{G}_{0}=S_{5} \times S^{1}$ and effective symmetry is $\mathfrak{H}_{0}=S_{4}$. Also, in such a case, (B.3) reduces to

$$
\left\{\begin{array}{l}
\dot{x}=f(x)+\alpha(3 x+y), \\
\dot{y}=f(y)+4 \alpha x .
\end{array}\right.
$$

Based on Example B.1, we make the following conjecture.

Conjecture B.1. Consider system (B.2). Suppose $\mathbf{S}(v)$ is a relative equilibrium with effective acting group $S_{m} \times S^{1}$ and effective symmetry $\mathbb{Z}_{m}^{t_{1}}$. Then, the corresponding reduced system is independent of $m$.

\section{Acknowledgments}

This article was supported by NSF through grant DMS-1413223. The first author is thankful to Guangzhou University for the support during Summer 2018.

\section{REFERENCES}

[1] R. Arkhipov, A. Amann, A. Vladimirov, Pulse repetition-frequency multiplication in a coupled cavity passively modelocked semiconductor lasers, Appl. Phys. 118 (2015), 539-548.

[2] R. Arkhipov, T. Habruseva, A. Pimenov, M. Radziunas, S. Hegarty, G. Huyet, A. Vladimirov, Semiconductor mode-locked lasers with coherent dual-mode optical injection: simulations, analysis, and experiment, J. Opt. Soc. Am. B 33 (2016), 351-359.

[3] R. Arkhipov, A. Pimenov, M. Radziunas, D. Rachinskii, A.G. Vladimirov, D. Arsenijević, H. Schmeckebier, D. Bimberg, Hybrid mode locking in semiconductor lasers: simulations, analysis, and experiments, IEEE J. Sel. Top. Quantum Electron. 19 (2013), $1100208 \mathrm{C} 1100208$.

[4] Z. Balanov, P. Kravetc, W. Krawcewicz, D. Rachinskii, Hopf Bifurcation of Relative Periodic Solutions: Case Study of a Ring of Passively Mode-Locked Lasers, ArXiv e-prints, March 2017. arXiv:1703.09154 [math.DS].

[5] Z. Balanov, W. Krawcewicz, Symmetric Hopf bifurcation: Twisted degree approach, In: Handbook of Differential Equations: Ordinary Differential Equations. Vol. 4, Elsevier, Amsterdam, 2008, 1-131. 
[6] Z. Balanov, W. Krawcewicz, D. Rachinskii, A. Zhezherun, Hopf bifurcation in symmetric networks of coupled oscillators with hysteresis, J. Dyn Differ. Equ. 24 (2012), 713-759.

[7] Z. Balanov, W. Krawcewicz, B. Rai, Taylor-couette problem and related topics, Nonlinear Anal. 4 (2003), 541-559, 2003.

[8] Z. Balanov, W. Krawcewicz, S. Rybicki, H. Steinlein, A short treatise on the equivariant degree theory and its applications, J. Fixed Point Theory Appl. 8 (2010), 1-74.

[9] Z. Balanov, W. Krawcewicz, H. Steinlein, Applied Equivariant Degree, vol. 1 of AIMS Series on Differential Equations \& Dynamical Systems, Springfield, Ill, USA, 2006.

[10] G.E. Bredon, Introduction to compact transformation groups, vol. 46, Academic press, 1972.

[11] T. Bröcker, T. Dieck, Representations of compact Lie groups, Graduate Texts in Mathematics 98, Springer, Berlin Heidelberg Germany, (2003).

[12] R. Broucke, On relative periodic solutions of the planar general three-body problem, Celestial Mechanics, 12 (1975), 439-462.

[13] S. Chandrasekhar, Ellipsoidal figures of equilibrium, vol. 9, Yale University Press, New Haven, 1969.

[14] P. Chossat, R. Lauterbach, Methods in equivariant bifurcations and dynamical systems, vol. 15, World Scientific Publishing Co Inc, 2000.

[15] T. Erneux, Applied delay differential equations, vol. 3, Springer, 2009.

[16] B. Fiedler, Global bifurcation of periodic solutions with symmetry, vol. 1309, Springer, 2006.

[17] B. Fiedler, S. Yanchuk, V. Flunkert, P. Hövel, H.J. Wünsche, E. Schöll, Delay stabilization of rotating waves near fold bifurcation and application to all-optical control of a semiconductor laser, Phys. Rev. E 77 (2008), 066207.

[18] M. Field, Equivariant dynamical systems, Tran. Amer. Math. Soc. 259 (1980), 185-205.

[19] M. Golubitsky, I. Stewart, The symmetry perspective: from equilibrium to chaos in phase space and physical space, vo. 200, Springer, 2003.

[20] R.L. Griess, Jr., Twelve sporadic groups, Springer Monographs in Mathematics, Springer-Verlag, Berlin, 1998.

[21] T. Habruseva, S.P. Hegarty, A.G. Vladimirov, A. Pimenov, D. Rachinskii, N. Rebrova, E. A. Viktorov, G. Huyet, Bistable regimes in an optically injected mode-locked laser, Opt. Express 20 (2012), 25572-25583.

[22] E. Hooton, Z. Balanov, W. Krawcewicz, D. Rachinskii, Sliding Hopf bifurcation in interval systems, Discrete Contin. Dyn. Syst. 37 (2017), 3545-3566.

[23] J. Ize, Equivariant degree, In Handbook of topological fixed point theory, pp. 301-337. Springer, 2005.

[24] J. Ize, A. Vignoli, Equivariant degree theory, vol. 8, Walter de Gruyter, 2003.

[25] L. Jaurigue, A. Pimenov, D. Rachinskii, E. Schöll, K. Lüdge, A.G. Vladimirov, Timing jitter of passively-mode-locked semiconductor lasers subject to optical feedback: A semi-analytic approach, Phys. Rev. A 92 (2015), 053807.

[26] A. Jechow, M. Lichtner, R. Menzel, M. Radziunas, D. Skoczowsky, A.G. Vladimirov, Stripe-array diode-laser in an off-axis external cavity: Theory and experiment, Opt. Express 17 (2009), 19599-19604.

[27] K. Kawakubo, The theory of transformation groups, Oxford University Press, 1991.

[28] I. Kozin, R. Roberts, J. Tennyson, Relative equilibria of $\mathrm{D}_{2} \mathrm{H}^{+}$and $\mathrm{H}_{2} \mathrm{D}^{+}$, Molecular Phys. 98 (2000), $295-307$.

[29] W. Krawcewicz, J. Wu, Theory of degrees, with applications to bifurcations and differential equations, vo. 17, WileyInterscience, 1997.

[30] M. Krupa, Bifurcations of relative equilibria, SIAM J. Math. Anal. 21 (1990), 1453-1486.

[31] J.S. Lamb, I. Melbourne, C. Wulff, Hopf bifurcation from relative periodic solutions; secondary bifurcations from meandering spirals, J. Difference Equ. Appl. 12 (2006), 1127-1145.

[32] R. Lang, K. Kobayashi, External optical feedback effects on semiconductor injection laser properties, IEEE J. Quantum Electronics, 16 (1980), 347-355.

[33] M. Lichtner, V.Z. Tronciu, A.G. Vladimirov, Theoretical investigation of striped and non-striped broad area lasers with off-axis feedback, IEEE J. Quantum Electronics 48 (2012), 353-360.

[34] J.E. Marsden, T.S. Ratiu, Introduction to Mechanics and Symmetry, 2nd ed., Texts in Applied Mathematics 17, Springer, New York, 1999.

[35] K. Meyer, G. Hall, D. Offin, Introduction to Hamiltonian dynamical systems and the N-body problem, vol. 90, Springer, 2008.

[36] K.R. Meyer, Periodic solutions of the N-body problem, vol. 1719, Springer, 1999. 
[37] J. Montaldi, R. Roberts, Relative equilibria of molecules, J. Nonlinear Sci. 9 (1999), 53-88.

[38] M. Nizette, D. Rachinskii, A. Vladimirov, M. Wolfrum, Pulse interaction via gain and loss dynamics in passive mode locking, Physica D 218 (2006), 95-104.

[39] S. Pekarsky, J.E. Marsden, Point vortices on a sphere: stability of relative equilibria, J. Math. 1 Phys. 39 (1998), $5894-5907$.

[40] E. Pérez-Chavela, S. Rybicki, Topological bifurcations of central configurations in the n-body problem, Nonlinear Anal. 14 (2013), 690-698.

[41] A. Pimenov, T. Habruseva, D. Rachinskii, S.P. Hegarty, G. Huyet, A.G. Vladimirov, Effect of dynamical instability on timing jitter in passively mode-locked quantum-dot lasers, Optics Lett. 39 (2014), 6815-6818.

[42] A. Pimenov, V.Z. Tronciu, U. Bandelow, A.G. Vladimirov, Dynamical regimes of a multistripe laser array with external off-axis feedback, J. Opt. Soc. Am. B 30 (2013), 1606-1613.

[43] A. Pimenov, E.A. Viktorov, S.P. Hegarty, T. Habruseva, G. Huyet, D. Rachinskii, A.G. Vladimirov, Bistability and hysteresis in an optically injected two-section semiconductor laser, Physical Rev. E 89 (2014), 052903.

[44] D. Puzyrev, A. Vladimirov, S. Gurevich, S. Yanchuk, Bound pulse trains in arrays of coupled spatially extended dynamical systems, Phys. Rev. Lett. 119 (2017), 163901.

[45] N. Rebrova, G. Huyet, D. Rachinskii, A.G. Vladimirov, Optically injected mode-locked laser, Physical Rev. E 83 (2011), 066202.

[46] S. Slepneva, B. Kelleher, B. OShaughnessy, S.P. Hegarty, A.G. Vladimirov, G. Huyet, Dynamics of Fourier domain modelocked lasers, Opt. Express 21 (2013), 19240-19251.

[47] T. tom Dieck, Transformation groups, vol. 8, Walter de Gruyter, 1987.

[48] A. Vanderbauwhede, M. Krupa, M. Golubitsky, Secondary bifurcations in symmetric systems, In Proc. Equadiff Conference, vol. 118, pp. 709-716. Lect. Notes in Pure \& Appl. Math, 1987.

[49] A.G. Vladimirov, G. Kozyreff, P. Mandel, Synchronization of weakly stable oscillators and semiconductor laser arrays, EPL (Europhys. Lett.) 61 (2003), 613.

[50] A.G. Vladimirov, D. Rachinskii, M. Wolfrum, Modeling of Passively Mode-Locked Semiconductor Lasers, pp. 183-216, Wiley-VCH Verlag GmbH \& Co. KGaA, 2012.

[51] A.G. Vladimirov, D. Turaev, Model for passive mode locking in semiconductor lasers, Physical Rev. A 72 (2005), 033808.

[52] A.G. Vladimirov, D. Turaev, G. Kozyreff, Delay differential equations for mode-locked semiconductor lasers, Opt. Lett. 29 (2004), 1221-1223.

[53] C. Wulff, M. Roberts, Hamiltonian systems near relative periodic orbits, SIAM J. Appl. Dyn. Syst. 1 (2012), 1-43.

[54] S. Yanchuk, K.R. Schneider, L. Recke, Dynamics of two mutually coupled semiconductor lasers: instantaneous coupling limit, Physical Rev. E 69 (2004), 056221.

[55] S. Yanchuk, J. Sieber, Relative equailibria and relative periodic solutions in systems with time-delay and $S^{1}$ symmetry, arXiv preprint arXiv:1306.3327, 2013. 